\title{
COMMUTATIVE SUBALGEBRAS OF THE RING OF DIFFERENTIAL OPERATORS ON A CURVE
}

\author{
P. Perkins
}

\begin{abstract}
Let $X$ denote an irreducible affine algebraic curve over the complex numbers. Let $\mathscr{O}(X)$ be the ring of regular functions on $X$. Denote by $\mathscr{D}(X)$ the ring of differential operators on $X$. We wish to characterize $\mathscr{O}(X)$ as a ring theoretic invariant of $\mathscr{D}(X)$. It is proved that $\mathscr{O}(X)$ equals the set of all locally ad-nilpotent elements of $\mathscr{D}(X)$ if and only if $X$ is not simply connected. However, for most simply connected curves, we show there exists a maximal commutative subalgebra of $\mathscr{D}(X)$, consisting of locally ad-nilpotent elements, which is not isomorphic to $\mathscr{O}(X)$.
\end{abstract}

0. Introduction. Let $X$ be a curve, that is, an irreducible affine algebraic curve over $\mathbb{C}$. Write $\mathscr{O}(X)$ for the ring of regular functions on $X$ and $\mathscr{D}(X)$ for the ring of differential operators on $X$. See [8] for the basic definitions and facts about rings of differential operators on curves. This paper is motivated by the following question. If $X$ and $Y$ are curves with $\mathscr{D}(X) \cong \mathscr{D}(Y)$, is $X \cong Y$ ? Write $\tilde{X}$ for the normalization of $X$. Stafford [9] considers this question for $X$ with $\tilde{X}=A^{1}$, the affine line. He shows that $\mathscr{D}(X) \cong \mathscr{D}(\tilde{X})$ if and only if $X=\tilde{X}$. He also shows that if $X$ is the cubic cusp $y^{2}=x^{3}$ and $\tilde{Y}=A^{1}$, then $X \cong Y$ if and only if $\mathscr{D}(X) \cong \mathscr{D}(Y)$. Higher dimensional non-isomorphic varieties can have isomorphic rings of differential operators, see Levasseur, Smith and Stafford [2].

If $u \in \mathscr{D}(X)$, define $\operatorname{ad}(u) \in \operatorname{End}_{\mathbb{C}}(\mathscr{D}(X))$ by $\operatorname{ad}(u)(v)=[u, v]=$ $u v-v u$. We say $u$ is locally ad-nilpotent if for every $v \in \mathscr{D}(X)$ there exists $n \in \mathbb{N}$ with $\operatorname{ad}(u)^{n}(v)=0$. Write

$$
\mathscr{N}(X)=\{u \in \mathscr{D}(X) \mid u \text { is locally ad-nilpotent }\} .
$$

Note that if $\vartheta: \mathscr{D}(Y) \rightarrow \mathscr{D}(X)$ is an isomorphism then $\vartheta(\mathscr{N}(Y))=$ $\mathscr{N}(X)$. It follows from the definition of $\mathscr{D}(X)$ that $\mathscr{O}(X)$ is a maximal commutative subalgebra of $\mathscr{D}(X)$ and that $\mathscr{O}(X)$ is contained in $\mathscr{N}(X)$. If genus $(X)>0$ then Makar-Limanov [3] shows that $\mathscr{O}(X)=\mathscr{N}(X)$. Hence if $\mathscr{D}(X) \cong \mathscr{D}(Y)$ with genus $(X)>0$ then $X \cong Y$.

This paper expands on Makar-Limanov's result to prove the following theorem. Let $\pi: \tilde{X} \rightarrow X$ denote the canonical surjection. 
THEOREM. Let $X$ be a curve. Then $\mathscr{N}(X)=\mathscr{O}(X)$ if and only if either

(i) $\tilde{X} \neq A^{1}$ or

(ii) $\tilde{X}=A^{1}$ and $\pi: \tilde{X} \rightarrow X$ is not injective.

CorollaRY 1. Let $X$ and $Y$ be curves with $\mathscr{D}(X) \cong \mathscr{D}(Y)$. If either (i) $\tilde{X} \neq \mathrm{A}^{1}$ or (ii) $\tilde{X}=\mathrm{A}^{1}$ and $\pi: \tilde{X} \rightarrow X$ is not injective, then $X \cong Y$.

The condition that $\tilde{X}=A^{1}$ and $\pi: \tilde{X} \rightarrow X$ is injective is equivalent to the condition that $X$ is simply connected. This observation is due to S. P. Smith and will be proved in $\S 5$. We obtain the following connection between algebra and topology.

Corollary 2. Let $X$ be a curve. Then $\mathscr{O}(X)=\mathscr{N}(X)$ if and only if $X$ is not simply connected. Thus if $\mathscr{D}(X) \cong \mathscr{D}(Y)$ and $X$ is not simply connected then $X \cong Y$.

If $\mathscr{O}(X) \neq \mathscr{N}(X)$ then $\tilde{X}=A^{1}$ and $\pi: \tilde{X} \rightarrow X$ is injective. In [8] it is shown, for such $X$, that $\mathscr{D}(X)$ is Morita equivalent to $\mathscr{D}\left(A^{1}\right)$. Thus if $X$ and $Y$ are curves with $\mathscr{O}(X) \neq \mathscr{N}(X)$ and $\mathscr{O}(Y) \neq \mathscr{N}(Y)$ then $\mathscr{D}(X)$ is Morita equivalent to $\mathscr{D}(Y)$. The results of [8] also show that $\mathscr{D}(X)$ and $\mathscr{D}(Y)$ are simple, hereditary $\mathbb{C}$-algebras with the same Krull and $G K$ dimensions. Hence it will be difficult to distinguish between them. Nevertheless, distinguishing between such "small" non-commutative algebras is an important problem in ring theory.

We will always write $\mathscr{D}\left(A^{1}\right)=\mathbb{C}[t, \partial]$ where $\partial=d / d t$. Dixmier [1] shows that all maximal commutative subalgebras of $\mathscr{D}\left(A^{1}\right)$, contained in $\mathscr{N}\left(A^{1}\right)$, are conjugate under Aut $\mathscr{D}\left(A^{1}\right)$. Thus if $\varphi: \mathscr{D}(X) \rightarrow \mathscr{D}\left(\mathrm{A}^{1}\right)$ is an isomorphism, there exists $\mu \in$ Aut $\mathscr{D}\left(A^{1}\right)$ such that $\mu \varphi$ restricts to an isomorphism between $\mathscr{O}(X)$ and $\mathbb{C}[t]=\mathscr{O}\left(\mathrm{A}^{1}\right)$. This leads one to ask, for an arbitrary curve $X$, if every maximal commutative subalgebra of $\mathscr{D}(X)$ which is contained in $\mathscr{N}(X)$ is isomorphic to $\mathscr{O}(X)$.

Let $X$ be a curve with $\tilde{X}=A^{1}$ and $\pi: \tilde{X} \rightarrow X$ injective. In $\S \S 3$ and 4 , we consider such curves with the additional hypothesis that $X$ has unique singularity at $\pi(0)$. It is shown that for all but one such curve, there are maximal commutative subalgebras of $\mathscr{D}(X)$ contained in $\mathscr{N}(X)$ but not isomorphic to $\mathscr{O}(X)$. The one "well behaved" curve is the cubic cusp $y^{2}=x^{3}$. Thus maximal commutative subalgebras of $\mathscr{D}(X)$ consisting of locally ad-nilpotent elements are not alone suffcient to distinguish these rings from one another. 
These results will appear in the author's Ph.D. thesis. I would like to thank my advisor, S. Paul Smith, for interesting me in these questions and for his many helpful comments and suggestions.

1. Curves with $\mathscr{O}(X)=\mathscr{N}(X)$. In this section it is proved that if $\mathscr{O}(X) \neq \mathscr{N}(X)$ then $\tilde{X}=\mathrm{A}^{1}$ and the canonical surjection $\pi: \tilde{X} \rightarrow X$ is injective. The rest of the paper considers the case with $\tilde{X}=A^{1}$ and $\pi$ injective; these are the only curves whose differential operator rings are hard to distinguish from one another. Theorem 1.1 and Corollary 1.2 are results of L. Makar-Limanov. We thank him for allowing us to present them here. The proofs will appear in [3]. Most of this section is based on his methods.

THEOREM 1.1 (Makar-Limanov). If $X$ is a curve and $\mathscr{O}(X) \neq \mathscr{N}(X)$ then $\mathscr{O}(X) \subset \mathbb{C}[b]$ for some $b$ transcendental over $\mathbb{C}$.

COROLLARY 1.2 (Makar-Limanov). If $X$ is a curve with genus greater than zero then $\mathscr{O}(X)=\mathscr{N}(X)$.

Using Theorem 1.1, we now show that if $\mathscr{O}(X) \neq \mathscr{N}(X)$ then $\mathscr{O}(\tilde{X})$ $=\mathbb{C}[t]$ for some $t$ transcendental over $\mathbb{C}$. Proposition 1.3 is probably well known but we could find no reference for it.

Proposition 1.3. Let $X$ be a curve with $\mathscr{O}(X) \subset \mathbb{C}[b]$ for some $b$ transcendental over $\mathbb{C}$. Then there exists $t \in \operatorname{Fract} \mathscr{O}(X)$ with $\mathscr{O}(\tilde{X})=$ $\mathbb{C}[t]$.

Proof. Recall that $\tilde{X}$ is an affine nonsingular curve. By Luroth's theorem, $\tilde{X}$ is rational, since Fract $\mathscr{O}(\tilde{X})=\operatorname{Fract} \mathscr{O}(X) \subset \mathbb{C}(b)$. Thus $\mathscr{O}(\tilde{X})$ is a UFD and every nonzero prime ideal of $\mathscr{O}(\tilde{X})$ is principal. Since $\mathscr{O}(X) \subset \mathbb{C}[b]$ and $\mathbb{C}[b]$ is integrally closed in $\mathbb{C}(b), \mathscr{O}(\tilde{X}) \subset \mathbb{C}[b]$. Choose $t \in \mathscr{O}(\tilde{X}) \backslash \mathbb{C}$ of minimal degree in $b$. Then for all $\alpha \in \mathbb{C}$, $t-\alpha$ generates a maximal ideal of $\mathscr{O}(\tilde{X})$. Indeed, if $(t-\alpha)$ were not maximal then $t-\alpha=x y$ with $x, y \in \mathscr{O}(\tilde{X})$ nonunits, since $\mathscr{O}(\tilde{X})$ is a UFD. But then $\operatorname{deg}_{b}(x)<\operatorname{deg}_{b}(t)$, a contradiction.

Let $\mathfrak{m}$ be the ideal generated by $t$. If $\mathfrak{m}^{\prime}$ is another maximal ideal and $\varphi: \mathscr{O}(\tilde{X}) \rightarrow \mathscr{O}(\tilde{X}) / \mathfrak{m}^{\prime}$, then $\varphi(t)=\alpha \in \mathbb{C}$. Thus $\mathfrak{m}^{\prime}$ contains the maximal ideal $(t-\alpha)$ and hence equals it. If $w$ is an irreducible element of $\mathscr{O}(\tilde{X})$ then $0 \neq(w)$ is prime and hence maximal since $\operatorname{dim} \mathscr{O}(\tilde{X})=1$. Thus $(w)=(t-\beta)$ for some $\beta \in \mathbb{C}$ and so $u w=t-\beta$ for some unit of $\mathscr{O}(\tilde{X})$. But the only units of $\mathscr{O}(\tilde{X})$ are in $\mathbb{C}$ since $\mathscr{O}(\tilde{X}) \subset \mathbb{C}[b]$. Hence, by unique factorization, any element of $\mathscr{O}(\tilde{X})$ is 
of the form $\lambda\left(t-\alpha_{1}\right) \cdots\left(t-\alpha_{k}\right)$ for $\lambda, \alpha_{1}, \ldots, \alpha_{k} \in \mathbb{C}$. Thus $\mathscr{O}(\tilde{X}) \subset \mathbb{C}[t]$ and hence there is equality.

CoRollary 1.4. If $X$ is a curve with $\mathscr{O}(X) \neq \mathscr{N}(X)$ then $\tilde{X}=\mathrm{A}^{1}$. Thus if $\tilde{X} \neq \mathrm{A}^{1}$ then $\mathscr{O}(X)=\mathscr{N}(X)$.

Proof. By Theorem 1.1, $\mathscr{O}(X) \subset \mathbb{C}[b]$. Proposition 1.3 implies $\mathscr{O}(\tilde{X})=\mathbb{C}[t]$ and this yields the result.

Corollary 1.5. Let $X$ and $Y$ be curves such that $\tilde{X} \neq A^{1}$. If $\mathscr{D}(X) \cong \mathscr{D}(Y)$ then $X \cong Y$.

If $\tilde{X}=\mathrm{A}^{1}$ it is still possible to have $\mathscr{O}(X)=\mathscr{N}(X)$. Proposition 1.8 clarifies the situation. First are some technical lemmas. If $\mathscr{O}(\tilde{X})=\mathbb{C}[t]$ then $\mathscr{D}(X) \subset \mathbb{C}(t)[\partial]$ and hence inherits the filtration by order of differential operator. We form the associated graded ring, denoted $\operatorname{gr}_{\partial} \mathscr{D}(X)$ to avoid confusion with another graded ring considered later in this paper. Write $\operatorname{gr}_{\partial} \mathscr{D}\left(A^{1}\right)=\mathbb{C}[t, \xi]$. If $\tilde{X}=A^{1}$ then $\operatorname{gr}_{\partial} \mathscr{D}(X) \subset$ $\mathbb{C}[t, \xi]$ by $[8,3.11]$. Throughout the paper we use $\{f, g\}$ to denote the Poisson bracket

$$
(\partial f / \partial \xi)(\partial g / \partial t)-(\partial f / \partial t)(\partial g / \partial \xi)
$$

with $f, g \in \mathbb{C}(t)[\xi]$. For $u, v \in \mathbb{C}(t)[\partial]$, it is straightforward to check that $\operatorname{gr}_{\partial}[u, v]=\left\{\operatorname{gr}_{\partial}(u), \operatorname{gr}_{\partial}(v)\right\}$ if the latter is nonzero. Thus $\left\{\operatorname{gr}_{\partial}(u), \cdot\right\}$ $\in \operatorname{End}_{\mathbb{C}}\left(\operatorname{gr}_{\partial} \mathscr{D}(X)\right)$ for all $u \in \mathscr{D}(X)$.

LEMMA 1.6. Let $p \xi^{n} \in \mathbb{C}[t, \xi]$ where $p \in \mathbb{C}[t]$ is of degree $r$ and $n>0$. Let $q \in \mathbb{C}[t]$ be of degree $s>0$. If $\delta=\left\{p \xi^{n}, \cdot\right\}$ is nilpotent on $q \xi^{m}$ then, for some $i>0, r m=n s+i(r-n)$.

Proof. Assume $r m \neq n s+i(r-n)$ for all $i>0$ and let $t^{r}$ be the leading term of $p$. We show by induction on $i$ that $\delta^{i}\left(q \xi^{m}\right)=q_{i} \xi^{m+i(n-1)}$ where $q_{i}$ has leading term $\alpha_{i} t^{s+i(r-1)}$ with $0 \neq \alpha_{i} \in \mathbb{C}$. This contradicts the hypothesis that $\delta$ is nilpotent on $q \xi^{m}$ and hence proves the lemma. Of course the result is true for $i=0$. Note that, if $h \in \mathbb{C}[t], \delta\left(h \xi^{l}\right)=$ $\left(n p h^{\prime}-l h p^{\prime}\right) \xi^{l+n-1}$ where $f^{\prime}$ denotes $d f / d t$. By induction, suppose $\delta^{i}\left(q \xi^{m}\right)=q_{i} \xi^{m+i(n-1)}$ where the leading term of $q_{i}$ is $\alpha_{i} t^{s+i(r-1)}$ with $0 \neq \alpha_{i} \in \mathbb{C}$. Then

$$
\delta^{i+1}\left(q \xi^{m}\right)=q_{i+1} \xi^{m+(i+1)(n-1)}
$$

and the leading term of $q_{i+1}$ is

$$
\alpha_{i}(n(s+i(r-1))-(m+i(n-1)) r) t^{s+(i+1)(r-1)} .
$$


Since $(n(s+i(r-1))-(m+i(n-1)) r)=n s+i(r-n)-r m \neq 0$ by hypothesis, this completes the proof.

LEMMA 1.7. If $X$ is a curve with $\tilde{X}=\mathrm{A}^{1}$ and $u \in \mathscr{N}(X) \backslash \mathscr{O}(X)$ then $\operatorname{gr}_{\partial}(u)=\lambda \xi^{n}$ for some positive integer $n$ and $\lambda \in \mathbb{C}$.

Proof. By way of contradiction, assume $\operatorname{gr}_{\partial}(u)=p \xi^{n}$ with $\operatorname{deg}(p)=$ $r>0$ and let $\operatorname{ann}_{\mathscr{O}(X)}(\mathbb{C}[t] / \mathscr{O}(X))=q \mathbb{C}[t]$. Then $q \mathbb{C}[t, \partial] \subset \mathscr{D}(X)$ and $q$ has order zero, whence $q \mathbb{C}[t, \xi] \subset \operatorname{gr}_{\partial} \mathscr{D}(X)$. Let $\operatorname{deg}(q)=s>0$ and assume $r \geq n$. Since $\left\{\operatorname{gr}_{\partial}(u), \cdot\right\}$ is nilpotent on $q$, Lemma 1.6 implies $0=r \cdot 0=n s+i(r-n) \geq s$, a contradiction. Thus $r<n$. Hence if $\left\{\operatorname{gr}_{\partial}(u), \cdot\right\}$ is nilpotent on $q \xi^{m}, r m=n s+i(r-n) \leq n s$. Choose $m$ such that $r m>n s$. Then $q \xi^{m} \in \operatorname{gr}_{\partial} \mathscr{D}(X)$ but $\left\{\operatorname{gr}_{\partial}(u), \cdot\right\}$ is not nilpotent on it. This contradiction implies $r=0$.

Proposition 1.8. If $X$ is a curve with $\tilde{X}=\mathrm{A}^{1}$ and $\mathscr{O}(X) \neq \mathscr{N}(X)$ then $\operatorname{dim}\left(\mathbb{C}[t, \xi] / \operatorname{gr}_{\partial} \mathscr{D}(X)\right)<\infty$. In particular, $\pi: \tilde{X} \rightarrow X$ is injective.

Proof. The second assertion follows from the first by [8, 3.12]. Choose $u \in \mathscr{N}(X) \backslash \mathscr{O}(X)$. By Lemma 1.7 we may assume that $\operatorname{gr}_{\partial}(u)=\xi^{n}$ with $n>0$. As in the proof of Lemma 1.7, pick $q \in$ $\mathbb{C}[t]$ such that $q \mathbb{C}[t, \xi] \subset \operatorname{gr}_{\partial} \mathscr{D}(X)$ and let $t^{s}$ be the leading term of $q$. We claim $\xi^{s(n-1)} \mathbb{C}[t, \xi] \subset \operatorname{gr}_{\partial} \mathscr{D}(X)$. Assuming the claim, let $v \in \mathbb{C}[t, \xi] \backslash \mathrm{gr}_{\partial} \mathscr{D}(X)$. Since $q \mathbb{C}[t, \xi] \subset \operatorname{gr}_{\partial} \mathscr{D}(X)$, we may assume $t-\operatorname{deg}(v) \leq s-1$. Since $\xi^{s(n-1)} \mathbb{C}[t, \xi] \subset \operatorname{gr}_{\partial} \mathscr{D}(X)$, we may assume $\xi-\operatorname{deg}(v) \leq s(n-1)-1$. Thus $v$ is in the finite dimensional vector space spanned by $\left\{t^{i} \xi^{j} \mid 0 \leq i \leq s-1,0 \leq j \leq s(n-1)-1\right\}$ and this proves the result.

To prove the claim, we show $t^{i} \xi^{j} \in \operatorname{gr}_{\partial} \mathscr{D}(X)$ for all $j \geq s(n-1)$ by induction on $i$. By the remarks before Lemma 1.6, $\delta=\left\{\xi^{n},-\right\} \in$ $\operatorname{End}\left(\operatorname{gr}_{\partial} \mathscr{D}(X)\right)$. Note that $\delta\left(f \xi^{m}\right)=n f^{\prime} \xi^{m+(n-1)}$ for $f \in \mathbb{C}[t]$. For all $m \geq 0, q \xi^{m} \in \mathrm{gr}_{\partial} \mathscr{D}(X)$. Hence so is $\delta^{s}\left(q \xi^{m}\right)=\alpha \xi^{m+s(n-1)}$ for some $0 \neq \alpha \in \mathbb{C}$. It follows that for all $j \geq s(n-1), \xi^{j} \in \operatorname{gr}_{\partial} \mathscr{D}(X)$ and we can start the induction. Assume that $i>0$ and for $k<i, j \geq s(n-1)$ implies that $t^{k} \xi^{j} \in \mathrm{gr}_{\partial} \mathscr{D}(X)$. If $i<s$ then

$$
\delta^{s-i}\left(q \xi^{l}\right)=\beta q^{(s-i)} \xi^{l+(s-i)(n-1)} \in \mathrm{gr}_{\partial} \mathscr{D}(X)
$$

for all $l \geq 0$, where $q^{(s-i)}$ denotes the $s-i$ th derivative of $q$ and $0 \neq \beta \in \mathbb{C}$. The leading term of $q^{(s-i)}$ is a nonzero constant times $t^{i}$ so our induction hypothesis implies that $t^{i} \xi^{l+(s-i)(n-1)} \in \operatorname{gr}_{\partial} \mathscr{D}(X)$ for all $l \geq i(n-1)$. If $i \geq s$ then $t^{i-s} \xi^{l} q \in \operatorname{gr}_{\partial} \mathscr{D}(X)$ for all $l \geq 0$. Since 
the leading term of $t^{i-s} \xi^{l} q$ is $t^{i} \xi^{l}$ and the induction hypothesis again applies, this completes the induction and the proof.

COROLlARY 1.9. If $X$ is a curve such that either

(i) $\tilde{X} \neq \mathrm{A}^{1}$ or

(ii) $\tilde{X}=A^{1}$ but $\pi: \tilde{X} \rightarrow X$ is not injective then $\mathscr{O}(X)=\mathscr{N}(X)$.

Proof. Combine Corollary 1.4 and Proposition 1.8.

COROLlaRY 1.10. Let $X$ and $Y$ be curves with $\mathscr{D}(X) \cong \mathscr{D}(Y)$. If either $\tilde{X} \neq \mathrm{A}^{1}$ or $\pi: \tilde{X} \rightarrow X$ is not injective then $X \cong Y$.

2. Locally Ad-nilpotent elements not in $\mathscr{O}(X)$. In $\S 1$ we saw that if either $\tilde{X} \neq \mathrm{A}^{1}$ or $\pi: \tilde{X} \rightarrow X$ is not injective then $\mathscr{O}(X)=\mathscr{N}(X)$. Thus in either of these two cases, $\mathscr{O}(X)$ is the unique maximal commutative subalgebra of $\mathscr{D}(X)$ contained in $\mathscr{N}(X)$. In this section we show, conversely, that if $\tilde{X}=A^{1}$ and $\pi: \tilde{X} \rightarrow X$ is injective then $\mathscr{N}(X)$ properly contains $\mathscr{O}(X)$. Indeed, $\mathscr{D}(X)$ contains a maximal commutative subalgebra $\mathscr{D}_{0}^{t}(X)$, consisting of locally ad-nilpotent elements, such that $\mathscr{D}_{0}^{t}(X) \cap \mathscr{O}(X)=\mathbb{C}$. Several of the results will be proved in a generality that will prove useful later.

When $X=A^{1}, \mathscr{D}_{0}^{t}(X)$ is just $\mathbb{C}[\partial]$ and in the general case $\mathscr{D}_{0}^{t}(X)$ should be thought of as analogous to $\mathbb{C}[\partial]$. However, in general there is no derivation $\delta \in \mathbb{D}(X)$ with $\mathbb{C}[\delta]$ a maximal commutative subalgebra of $\mathscr{D}(X)$ contained in $\mathscr{N}(X)$. Thus finding the correct analogy of $\mathbb{C}[\partial]$ is complicated.

Throughout $\S 2$, let $X$ be a curve with $\tilde{X}=A^{1}$.

Definition. Let $R$ be a subalgebra of $\mathbb{C}(t)$ [ $\partial]$. A commutative subalgebra $S$ of $R$ is ad-nilpotent if, for every $u \in R$, there exists $n \in \mathbb{N}$ with $\left[s_{0},\left[s_{1}, \ldots\left[s_{n}, u\right]\right]\right]=0$ for all $s_{0}, \ldots, s_{n} \in S$. An ad-nilpotent subalgebra of $\mathscr{D}(X)$ is always contained in $\mathscr{N}(X)$. By the definition of $\mathscr{D}(X), \mathscr{O}(X)$ is an ad-nilpotent subalgebra of $\mathscr{D}(X)$.

Let $K=\mathbb{C}(t)$ and consider $\mathscr{D}(K)=\mathbb{C}(t)[\partial]$. An element $u \in \mathscr{D}(K)$ can be uniquely expressed $u=\sum f_{k} \partial^{k}$ where $k$ ranges over a finite subset of $\mathbb{N}$ and $f_{k} \in \mathbb{C}(t)$. Since $\tilde{X}=A^{1}$, we may identify $\mathscr{D}(X)$ with a subalgebra of $\mathscr{D}(K)$. Set $\operatorname{deg}(f / g)=\operatorname{deg}(f)-\operatorname{deg}(g)$ for $f, g \in \mathbb{C}[t]$.

Definition. If $u=\sum f_{k} \partial^{k} \in \mathscr{D}(K)$ define the $t$-degree of $u$ to be $t-\operatorname{deg}(u)=\max \left\{\operatorname{deg}\left(f_{k}\right)\right\}$. Set $\mathscr{D}_{k}^{t}(K)=\{u \in \mathscr{D}(K) \mid t-$ $\operatorname{deg}(u) \leq k\}$ for $k \in \mathbb{Z}$. It is clear that $\mathscr{D}_{i}^{t}(K) \cdot \mathscr{D}_{j}^{t}(K) \subset \mathscr{D}_{i+j}^{t}(K)$ 
so we obtain the filtration $\mathscr{D}(K)=\bigcup_{k \in \mathbb{Z}} \mathscr{D}_{k}^{t}(K)$. Form the graded ring $\bigoplus_{k \in \mathbb{Z}}\left(\mathscr{D}_{k}^{t}(K) / \mathscr{D}_{k-1}^{t}(K)\right)$ in the usual way and denote this ring $\mathrm{gr}_{t} \mathscr{D}(K)$. Define the $t$-filtration on $\mathscr{D}(X)$ by $\mathscr{D}_{k}^{t}(X)=\mathscr{D}(X) \cap \mathscr{D}_{k}^{t}(K)$. Then $\mathrm{gr}_{t} \mathscr{D}(X)$ embeds in $\mathrm{gr}_{t} \mathscr{D}(K)$.

Lemma 2.1. For all $n, m \in \mathbb{Z},\left[\mathscr{D}_{n}^{t}(K), \mathscr{D}_{m}^{t}(K)\right] \subset \mathscr{D}_{n+m-1}^{t}(K)$ and hence $\operatorname{~gr}_{t} \mathscr{D}(K)$ is commutative.

Proof. Let $h, f, p, q \in \mathbb{C}[t]$, with $\operatorname{deg}(h / f)=n$ and $\operatorname{deg}(p / q)=m$. If $k, l \in \mathbb{N}$, it suffices to prove $\left[(h / f) \partial^{k},(p / q) \partial^{l}\right] \in \mathscr{D}_{n+m-1}^{t}(K)$. Now

$$
\left[(h / f) \partial^{k},(p / q) \partial^{l}\right]=(h / f)\left[\partial^{k}, p / q\right] \partial^{l}+(p / q)\left[h / f, \partial^{l}\right] \partial^{k} .
$$

But

$$
\begin{aligned}
{\left[h / f, \partial^{l}\right] } & =-\sum_{0 \leq i \leq l-1} \partial^{i}[\partial, h / f] \partial^{l-i-1} \\
& =-\sum_{0 \leq i \leq l-1} \partial^{i}\left(f h^{\prime}-h f^{\prime}\right) f^{-2} \partial^{l-i-1}
\end{aligned}
$$

so $t-\operatorname{deg}\left[h / f, \partial^{l}\right] \leq \operatorname{deg}\left(f h^{\prime}-h f^{\prime}\right) f^{-2} \leq n-1$. Thus $(h / f)\left[\partial^{k}, p / q\right] \partial^{l}$ and $(p / q)\left[h / f, \partial^{l}\right] \partial^{k}$ are in $\mathscr{D}_{n+m-1}^{t}(K)$ and the result follows.

\section{LEMMA 2.2. $\operatorname{gr}_{t} \mathscr{D}(K) \cong \mathbb{C}\left[s, s^{-1}\right][\xi]$.}

Proof. Since $\mathrm{gr}_{t} \mathscr{D}(K)$ is commutative and $\operatorname{gr}_{t}(t) \mathrm{gr}_{t}\left(t^{-1}\right)=1$, there exists an algebra homomorphism

$$
\varphi: \mathbb{C}\left[s, s^{-1}\right][\xi] \rightarrow \operatorname{gr}_{t} \mathscr{D}(K)
$$

given by $\varphi(s)=\operatorname{gr}_{t}(t), \varphi\left(s^{-1}\right)=\operatorname{gr}_{t}\left(t^{-1}\right)$ and $\varphi(\xi)=\operatorname{gr}_{t}(\partial)$. If $\varphi\left(\sum f_{i}(\xi) s^{i}\right)=0$ then $\sum \operatorname{gr}_{t}\left(f_{i}(\partial) t^{i}\right)=0$, whence $f_{i}(\partial)=0$ for all $i$. Thus $\varphi$ is injective. If $f \in K$ then $f=g / h$ with $g, h \in \mathbb{C}[t]$. Write $g=\alpha t^{n}+p$ and $h=\beta t^{m}+q$ where $\alpha, \beta \neq 0, \operatorname{deg}(p)<n$ and $\operatorname{deg}(q)<m$. Now $f, \alpha \beta^{-1} t^{n-m} \in \mathscr{D}_{n-m}^{t}(K)$ and

$$
\begin{aligned}
f-\alpha \beta^{-1} t^{n-m} & =\left(g-\alpha \beta^{-1} t^{n-m} h\right) / h \\
& =\left(p-\alpha \beta^{-1} t^{n-m} q\right) / h \in \mathscr{D}_{n-m-1}^{t}(K) .
\end{aligned}
$$

Thus $\operatorname{gr}_{t}(f)=\operatorname{gr}_{t}\left(\alpha \beta^{-1} t^{n-m}\right) \in \operatorname{Im}(\varphi)$. If $u \in \mathscr{D}(K)$ write $u=\sum f_{i} \partial^{i}$ with $f_{i} \in K$ and set $t-\operatorname{deg}(u)=k$. If $a, b \in \mathscr{D}(K)$ such that $t-$ $\operatorname{deg}(a+b)=t-\operatorname{deg}(a)=t-\operatorname{deg}(b)$ then $\operatorname{gr}_{t}(a+b)=\operatorname{gr}_{t}(a)+\operatorname{gr}_{t}(b)$. It follows that

$$
\operatorname{gr}_{t}(u)=\operatorname{gr}_{t}\left(\sum\left\{f_{i} \partial^{i} \mid \operatorname{deg}\left(f_{i}\right)=k\right\}\right)=\sum\left\{\operatorname{gr}_{t}\left(f_{i} \partial^{i}\right) \mid \operatorname{deg}\left(f_{i}\right)=k\right\}
$$

and each $\operatorname{gr}_{t}\left(f_{i} \partial^{i}\right)=\operatorname{gr}_{t}\left(f_{i}\right) \operatorname{gr}_{t}(\partial)^{i} \in \operatorname{Im}(\varphi)$. Thus $\varphi$ is surjective. 
If $R$ is any subalgebra of $\mathscr{D}(K)$, define the $t$-filtration on $R$ any $R_{n}^{t}=R \cap \mathscr{D}_{n}^{t}(K)$. We now prove the basic results about $R_{0}^{t}$.

Lemma 2.3. Let $R$ be a subalgebra of $\mathscr{D}(K)$ such that $\mathrm{gr}_{t}(R) \subset$ $\mathbb{C}[s, \xi]$ and let $v \in R_{0}^{t}$. Let $k>0$ and $u \in \mathscr{D}(K)$. If $v \notin \mathbb{C}$ then $[v, u] \in \mathscr{D}_{k-1}^{t}(K)$ implies $u \in \mathscr{D}_{k}^{t}(K)$.

Proof. Assume there exists $n>k$ with $u \in \mathscr{D}_{n}^{t}(K) \backslash \mathscr{D}_{n-1}^{t}(K)$. Write $u=t^{n} f(\partial)+x$ with $x \in \mathscr{D}_{n-1}^{t}(K)$. Write $v=g(\partial)+y \in \mathscr{D}_{0}^{t}(K)$ with $y \in \mathscr{D}_{-1}^{t}(K)$. Since $v \notin \mathbb{C}, \operatorname{deg}(g)>0$ because $\operatorname{gr}_{t}(R) \subset \mathbb{C}[s, \xi]$. Hence

$$
\begin{aligned}
{[v, u] } & =\left[g(\partial), t^{n} f(\partial)\right]+[y, u]+[g(\partial), x] \\
& =n t^{n-1} f(\partial) g^{\prime}(\partial)+\left(\text { something in } \mathscr{D}_{n-2}^{t}(K)\right) .
\end{aligned}
$$

Thus $[v, u] \notin \mathscr{D}_{k-1}^{t}(K)$.

By $[8,3.11], \operatorname{gr}_{\partial} \mathscr{D}(X) \subset \operatorname{gr}_{\partial} \mathscr{D}(\tilde{X})=\mathbb{C}[t, \xi]$ so we may take $R=$ $\mathscr{D}(X)$ in the following result.

Proposition 2.4. Let $R$ be a subalgebra of $\mathscr{D}(K)$ such that $\operatorname{gr}_{\partial}(R) \subset$ $\mathbb{C}[t, \xi]$. Then

(a) $\operatorname{gr}_{t}(R) \subset \mathbb{C}[s, \xi]$.

(b) $R_{0}^{t}$ is a commutative ad-nilpotent subalgebra of $R$.

(c) $R_{0}^{t} \cong \mathrm{gr}_{t}(R) \cap \mathbb{C}[\xi]$ as $\mathbb{C}$-algebras.

(d) If $R_{0}^{t} \neq \mathbb{C}$ then it is a maximal commutative subalgebra of $R$.

Proof. (a) If $u \in R$ with $t-\operatorname{deg}(u)<0$ then $u=\sum f_{k} \partial^{k}$ with $\operatorname{deg}\left(f_{k}\right)<0$ for all $k$, whence $\operatorname{gr}_{\partial}(u) \notin \mathbb{C}[t, \xi]$. Hence, if $u \in R$ then $t-\operatorname{deg}(u)=n \geq 0$. Write $u=t^{n} h(\partial)+v$ with $v \in \mathscr{D}_{n-1}^{t}(K)$ and $h$ a polynomial. Then $\operatorname{gr}_{t}(u)=s^{n} h(\xi) \in \mathbb{C}[s, \xi]$.

(b) Since elements of $R$ have non-negative $t$-degrees, $R_{-1}^{t}=(0)$. By Lemma $2.1,\left[R_{0}^{t}, R_{n}^{t}\right] \subset R_{n-1}^{t}$. Thus $R_{0}^{t}$ is ad-nilpotent. It is commutative because $\left[R_{0}^{t}, R_{0}^{t}\right] \subset R_{-1}^{t}=(0)$.

(c) Note that $\mathrm{gr}_{t} R \cap \mathbb{C}[\xi]$ is the image of $R_{0}^{t}$ under the map $R \rightarrow$ $\operatorname{gr}_{t}(R)$. But this image is isomorphic to $\operatorname{gr}_{t}\left(R_{0}^{t}\right)$ under the induced filtration. Since $R_{-1}^{t}=(0)$,

$$
\mathrm{gr}_{t}(R) \cap \mathbb{C}[\xi] \cong \mathrm{gr}_{t}\left(R_{0}^{t}\right) \cong R_{0}^{t} / R_{-1}^{t} \cong R_{0}^{t}
$$

as $\mathbb{C}$-algebras.

(d) Let $v \in R_{0}^{t} \backslash\{\mathbb{C}\}$ and $u \in R$. By (a), $\operatorname{gr}_{t}(R) \subset \mathbb{C}[s, \xi]$. Thus if $[v, u]=0 \in R_{-1}^{t}$ then $u \in R_{0}^{t}$ by Lemma 2.3. 
By Proposition 2.4 and the remark before it, $\operatorname{gr}_{t} \mathscr{D}(X) \subset \mathbb{C}[s, \xi]$. Thus if $\mathscr{D}_{0}^{t}(X) \neq \mathbb{C}$, Lemma 2.3 implies that the $t$-filtration on $\mathscr{D}(X)$ can be defined by the degree of ad-nilpotence of $\mathscr{D}_{0}^{t}(X)$ on $\mathscr{D}(X)$. That is

$\mathscr{D}_{n}^{t}(X)$

$=\left\{u \in \mathscr{D}(X) \mid\left[v_{0},\left[v_{1}, \ldots\left[v_{n}, u\right] \ldots\right]\right]=0\right.$ for all $\left.v_{0}, \ldots, v_{n} \in \mathscr{D}_{0}^{t}(X)\right\}$.

This is analogous to the way the order of operator filtration is defined, with $\mathscr{D}_{0}^{t}(X)$ playing the role of $\mathscr{O}(X)$.

Our next goal is to prove $\mathscr{D}_{0}^{t}(X) \neq \mathbb{C}$, whence it is a maximal commutative ad-nilpotent subalgebra. This will be achieved in Theorem 2.7. Note that $\operatorname{gr}_{t} \mathscr{D}\left(\mathrm{A}^{1}\right)=\mathbb{C}[s, \xi]$ and let $M \subset N$ be distinct nonzero $\operatorname{gr}_{t} \mathscr{D}\left(\mathrm{A}^{1}\right)$-submodules of $\mathrm{gr}_{t} \mathscr{D}(K)$. The 1-length of $N / M$ is defined, as in $[8,3.10]$, to be the largest integer $n$ such that there exists a chain of $\mathrm{gr}_{t} \mathscr{D}\left(\mathrm{A}^{1}\right)$-modules $M=M_{0} \subset M_{1} \subset \cdots \subset M_{n}=N$ with $\operatorname{dim}_{\mathbb{C}}\left(M_{i} / M_{i-1}\right)=\infty$ for each $i$.

LEMMA 2.5. Let $q \in \mathbb{C}[t]$. (i) If $P$ is a left $\mathscr{D}\left(A^{1}\right)$-module and $\mathscr{D}\left(\mathrm{A}^{1}\right) q^{-1} \supset P \supset \mathscr{D}\left(\mathrm{A}^{1}\right)$ then

$$
\text { 1-length }\left(\mathrm{gr}_{t} P / \mathrm{gr}_{t} \mathscr{D}\left(\mathrm{A}^{1}\right)\right)=\text { length }_{\mathscr{D}\left(\mathrm{A}^{1}\right)}\left(P / \mathscr{D}\left(\mathrm{A}^{1}\right)\right) \text {. }
$$

(ii) If $P$ is a right $\mathscr{D}\left(\mathrm{A}^{1}\right)$-module and $\mathscr{D}\left(\mathrm{A}^{1}\right) \supset P \supset q \mathscr{D}\left(\mathrm{A}^{1}\right)$ then

$$
\text { 1-length }\left(\mathrm{gr}_{t} \mathscr{D}\left(\mathrm{A}^{1}\right) / \mathrm{gr}_{t} P\right)=\text { length }_{\mathscr{D}\left(\mathrm{A}^{1}\right)}\left(\mathscr{D}\left(\mathrm{A}^{1}\right) / P\right) \text {. }
$$

Proof. Let the leading term of $q$ be $t^{k}$. We claim

$$
\begin{aligned}
\text { 1-length } & \left(\mathrm{gr}_{t} \mathscr{D}\left(\mathrm{A}^{1}\right) / \mathrm{gr}_{t}\left(q \mathscr{D}\left(\mathrm{A}^{1}\right)\right)\right) \\
& =\text { length }_{\mathscr{D}\left(\mathrm{A}^{1}\right)}\left(\mathscr{D}\left(\mathrm{A}^{1}\right) / q \mathscr{D}\left(\mathrm{A}^{1}\right)\right)
\end{aligned}
$$

and

$$
\begin{aligned}
\text { 1-length } & \left(\mathrm{gr}_{t}\left(\mathscr{D}\left(\mathrm{A}^{1}\right) q^{-1}\right) / \mathrm{gr}_{t} \mathscr{D}\left(\mathrm{A}^{1}\right)\right) \\
& =\text { length }_{\mathscr{D}\left(\mathbf{A}^{1}\right)}\left(\mathscr{D}\left(\mathrm{A}^{1}\right) q^{-1} / \mathscr{D}\left(\mathrm{A}^{1}\right)\right) .
\end{aligned}
$$

To prove the claim, note that

$$
\begin{aligned}
\text { length }_{\mathscr{D}\left(\mathrm{A}^{1}\right)} & \left(\mathscr{D}\left(\mathrm{A}^{1}\right) / q \mathscr{D}\left(\mathrm{A}^{1}\right)\right) \\
\quad & =\text { length }_{\mathscr{D}\left(\mathrm{A}^{1}\right)}\left(\mathscr{D}\left(\mathrm{A}^{1}\right) q^{-1} / \mathscr{D}\left(\mathrm{A}^{1}\right)\right)=k .
\end{aligned}
$$

If $u=q v \in q \mathscr{D}\left(\mathrm{A}^{1}\right)$ then

$$
\operatorname{gr}_{t}(u)=\left(\operatorname{gr}_{t}(q)\right)\left(\operatorname{gr}_{t}(v)\right)=s^{k}\left(\operatorname{gr}_{t}(v)\right) \in s^{k} \mathbb{C}[s, \xi] .
$$


Thus to see $s^{k} \mathbb{C}[s, \xi]=\operatorname{gr}_{t}\left(q \mathscr{D}\left(A^{1}\right)\right)$, it suffices to note

$$
s^{k+i} \xi^{j}=\operatorname{gr}_{t}\left(q t^{i} \partial^{j}\right) \in \operatorname{gr}_{t}\left(q \mathscr{D}\left(\mathrm{A}^{1}\right)\right)
$$

for all $i, j \in \mathbb{N}$. The first part of the claim follows from

$$
k=1 \text {-length }\left(\mathbb{C}[s, \xi] / s^{k} \mathbb{C}[s, \xi]\right) .
$$

A similar proof, using $\operatorname{gr}_{t}\left(q^{-1}\right)=s^{-k}$, shows

$$
\operatorname{gr}_{t}\left(\mathscr{D}\left(A^{1}\right) q^{-1}\right)=s^{-k} \mathbb{C}[s, \xi] .
$$

Since $k=\operatorname{deg}(q)=1$-length $\left(s^{-k} \mathbb{C}[s, \xi] / \mathbb{C}[s, \xi]\right)$, the second part also follows.

Note that if $L \supset N$ are right $\mathscr{D}\left(A^{1}\right)$-submodules of $\mathscr{D}(K)$ then

$$
\text { length }_{\mathscr{D}\left(\mathrm{A}^{\prime}\right)}(L / N) \leq 1 \text {-length }\left(\mathrm{gr}_{t} L / \mathrm{gr}_{t} N\right) \text {. }
$$

The result now follows just as in [8, 3.10]. By the above paragraph and the inequality $(*)$ we have

$$
\begin{aligned}
\text { length }_{\mathscr{D}\left(\mathrm{A}^{1}\right)}\left(\mathscr{D}\left(\mathrm{A}^{1}\right) / q \mathscr{D}\left(\mathrm{A}^{1}\right)\right) \\
\quad=1 \text {-length }\left(\mathrm{gr}_{t} \mathscr{D}\left(\mathrm{A}^{1}\right) / \mathrm{gr}_{t}\left(q \mathscr{D}\left(\mathrm{A}^{1}\right)\right)\right) \\
\quad=1 \text {-length }\left(\mathrm{gr}_{t} \mathscr{D}\left(\mathrm{A}^{1}\right) / \mathrm{gr}_{t} P\right)+1 \text {-length }\left(\mathrm{gr}_{t} P / \mathrm{gr}_{t}\left(q \mathscr{D}\left(\mathrm{A}^{1}\right)\right)\right) \\
\quad \geq \text { length }_{\mathscr{D}\left(\mathrm{A}^{1}\right)}\left(\mathscr{D}\left(\mathrm{A}^{1}\right) / P\right)+\operatorname{length}_{\mathscr{D}\left(\mathrm{A}^{1}\right)}\left(P / q \mathscr{D}\left(\mathrm{A}^{1}\right)\right) \\
\quad=\text { length }_{\mathscr{D}\left(\mathrm{A}^{1}\right)}\left(\mathscr{D}\left(\mathrm{A}^{1}\right) / q \mathscr{D}\left(\mathrm{A}^{1}\right)\right) .
\end{aligned}
$$

Thus

$$
\begin{aligned}
& \text { 1-length }\left(\mathrm{gr}_{t} \mathscr{D}\left(\mathrm{A}^{1}\right) / \mathrm{gr}_{t} P\right)+1 \text {-length }\left(\mathrm{gr}_{t} P / \mathrm{gr}_{t}\left(q \mathscr{D}\left(\mathrm{A}^{1}\right)\right)\right) \\
& \quad=\operatorname{length}_{\mathscr{D}\left(\mathrm{A}^{1}\right)}\left(\mathscr{D}\left(\mathrm{A}^{1}\right) / P\right)+\operatorname{length}_{\mathscr{D}\left(\mathrm{A}^{\prime}\right)}\left(P / q \mathscr{D}\left(\mathrm{A}^{1}\right)\right) .
\end{aligned}
$$

By $(*)$,

$$
\text { 1-length }\left(\mathrm{gr}_{t} \mathscr{D}\left(\mathrm{A}^{1}\right) / \mathrm{gr}_{t} P\right) \geq \text { length }_{\mathscr{D}\left(\mathrm{A}^{1}\right)}\left(\mathscr{D}\left(\mathrm{A}^{1}\right) / P\right)
$$

and

$$
\text { 1-length }\left(\operatorname{gr}_{t} P / \operatorname{gr}_{t}\left(q \mathscr{D}\left(A^{1}\right)\right)\right) \geq \text { length }_{\mathscr{D}\left(\mathrm{A}^{1}\right)}\left(P / q \mathscr{D}\left(\mathrm{A}^{1}\right)\right)
$$

so 1-length $\left(\mathrm{gr}_{t} \mathscr{D}\left(\mathrm{A}^{1}\right) / \mathrm{gr}_{t} P\right)=$ length $_{\mathscr{D}\left(\mathbf{A}^{1}\right)}\left(\mathscr{D}\left(\mathrm{A}^{1}\right) / P\right)$, proving (ii). The proof of $(\mathrm{i})$ is similar.

LEMMA 2.6. Let $R$ be a subalgebra of $\mathscr{D}(K)$ with $\operatorname{gr}_{\partial} R \subset \mathbb{C}[t, \xi]$. Assume there exist a left $\mathscr{D}\left(\mathrm{A}^{1}\right)$-module $P$, a right $\mathscr{D}\left(\mathrm{A}^{1}\right)$-module $Q$, and $q \in \mathbb{C}[t]$ with the following properties.

(i) $\mathscr{D}\left(\mathrm{A}^{1}\right) q^{-1} \supset P \supset \mathscr{D}\left(\mathrm{A}^{1}\right) \supset Q \supset q \mathscr{D}\left(\mathrm{A}^{1}\right)$, 
(ii) length $\left(P / \mathscr{D}\left(A^{1}\right)\right)=$ length $\left(\mathscr{D}\left(A^{1}\right) / Q\right)$,

(iii) $Q P \subset R$.

Then $\operatorname{gr}_{t} R \subset \mathbb{C}[s, \xi]$ and $\operatorname{dim}_{\mathbb{C}}\left(\mathbb{C}[s, \xi] / \operatorname{gr}_{t} R\right)<\infty$.

Proof. Proposition 2.4 implies $\operatorname{gr}_{t} R \subset \mathbb{C}[s, \xi]$. The rest of the proof is very similar to the proof of $[8,3.12]$. We have

$$
\mathbb{C}[s, \xi] \supset \mathrm{gr}_{t} R \supset \mathrm{gr}_{t}(Q P) \supset \mathrm{gr}_{t}(Q) \mathrm{gr}_{t}(P) .
$$

By Lemma 2.5,

(**) 1-length $\left(\operatorname{gr}_{t} P / \operatorname{gr}_{t} \mathscr{D}\left(A^{1}\right)\right)=$ length $\left(P / \mathscr{D}\left(A^{1}\right)\right)$

$$
=\operatorname{length}\left(\mathscr{D}\left(A^{1}\right) / Q\right)=1 \text {-length }\left(\operatorname{gr}_{t} \mathscr{D}\left(A^{1}\right) / \operatorname{gr}_{t} Q\right) \text {. }
$$

Set this common length equal to $m$. Note that if $I_{1} \subset I_{2}$ and $J$ are nonzero ideals of $\mathbb{C}[s, \xi]$ with $\operatorname{dim}_{\mathbb{C}}\left(I_{2} / I_{1}\right)=\infty$, then $\operatorname{dim}_{\mathbb{C}}\left(J I_{2} / J I_{1}\right)=$ $\infty$ also. Thus, if $\mathbb{C}[s, \xi]=I_{0} \subset I_{1} \subset \cdots \subset I_{m}=\operatorname{gr}_{t} P$ is a maximal chain for which $\operatorname{dim}_{\mathbb{C}}\left(I_{j} / I_{j-1}\right)=\infty$ for all $j$, then

$$
\mathrm{gr}_{t} Q=\left(\mathrm{gr}_{t} Q\right) I_{0} \subset \cdots \subset\left(\mathrm{gr}_{t} Q\right) I_{m}=\left(\mathrm{gr}_{t} Q\right)\left(\mathrm{gr}_{t} P\right)
$$

is also a chain for which each factor is infinite dimensional. Hence

$$
\text { 1-length }\left(\left(\mathrm{gr}_{t} Q\right)\left(\mathrm{gr}_{t} P\right) / \mathrm{gr}_{t} Q\right) \geq 1 \text {-length }\left(\mathrm{gr}_{t} P / \mathbb{C}[s, \xi]\right)
$$

But

$$
\begin{aligned}
& \text { 1-length }\left(\mathrm{gr}_{t} P / \mathbb{C}[s, \xi]\right)=1 \text {-length }\left(\mathbb{C}[s, \xi] / \mathrm{gr}_{t} Q\right) \\
& \geq 1 \text {-length }\left(\left(\mathrm{gr}_{t} Q\right)\left(\mathrm{gr}_{t} P\right) / \mathrm{gr}_{t} Q\right)
\end{aligned}
$$

by $(* *)$, whence

$$
\text { 1-length }\left(\mathbb{C}[s, \xi] / \operatorname{gr}_{t} Q\right)=1 \text {-length }\left(\left(\operatorname{gr}_{t} Q\right)\left(\operatorname{gr}_{t} P\right) / \mathrm{gr}_{t} Q\right) \text {. }
$$

Thus $\operatorname{dim}_{\mathbb{C}}\left(\mathbb{C}[s, \xi] /\left(\operatorname{gr}_{t} Q\right)\left(\operatorname{gr}_{t} P\right)\right)<\infty$ and this proves the result.

If $X$ and $Y$ are any pair of birationally equivalent curves then let $K=\operatorname{Fract} \mathscr{O}(X)=\operatorname{Fract} \mathscr{O}(Y)$ and define

$$
\mathscr{D}(X, Y)=\{D \in \mathscr{D}(K) \mid D * f \in \mathscr{O}(Y) \text { for all } f \in \mathscr{O}(X)\},
$$

where $D * f$ denotes the action of $D$ on $f$. If $X$ and $Y$ are curves such that $\mathscr{O}(X) \subset \mathscr{O}(Y) \subset(\tilde{X})$ then $\mathscr{D}(Y, X)$ is a left ideal of $\mathscr{D}(X)$ and a right ideal of $\mathscr{D}(Y)$. Note that $1 \in \mathscr{D}(X, Y)$ and $\mathscr{D}(Y, X) \cap \mathscr{O}(Y)$ equals the conductor of $\mathscr{O}(Y)$ in $\mathscr{O}(X)$, whence both $\mathscr{D}(X, Y)$ and $\mathscr{D}(Y, X)$ are nonzero. If $\pi: \tilde{X} \rightarrow X$ is injective then $\mathscr{D}(\tilde{X}, X) \mathscr{D}(X, \tilde{X})=\mathscr{D}(X)$ since it is a nonzero 2-sided ideal of a simple ring, by [8, 3.4]. Similarly $\mathscr{D}(X, \tilde{X}) \mathscr{D}(\tilde{X}, X)=\mathscr{D}(\tilde{X})$. 
TheOREM 2.7. Let $\tilde{X}=\mathrm{A}^{1}$ and $\pi: \tilde{X} \rightarrow X$ be injective. Then

(a) $\mathscr{D}_{0}^{t}(X)$ is a maximal commutative ad-nilpotent subalgebra of $\mathscr{D}(X)$.

(b) $\mathscr{D}_{0}^{t}(X) \cap \mathscr{O}(X)=\mathbb{C}$.

(c) $\mathscr{D}_{0}^{t}(X)$ is a finitely generated $\mathbb{C}$-domain of dimension 1 with integral closure isomorphic to $\mathbb{C}[\xi]$.

Proof. As remarked earlier, $\operatorname{gr}_{\partial} \mathscr{D}(X) \subset \mathbb{C}[t, \xi]$ by [8, 3.11]. Thus Proposition 2.4 applies and $\mathscr{D}_{0}^{t}(X)$ is a commutative ad-nilpotent subalgebra of $\mathscr{D}(X)$ intersecting $\mathscr{O}(X)$ in $\mathbb{C}$. Furthermore, to see that it is a maximal commutative subalgebra of $\mathscr{D}(X)$, it is enough to show $\mathscr{D}_{0}^{t}(X) \neq \mathbb{C}$. Thus it suffices to prove (c).

We do this by applying Lemma 2.6 to $P=\mathscr{D}(\tilde{X}, X)$ and $P^{*}=$ $\mathscr{D}(X, \tilde{X})$. Note that $\mathrm{gr}_{t} \mathscr{D}(X) \supset\left(\operatorname{gr}_{t}(P)\right)\left(\operatorname{gr}_{t}\left(P^{*}\right)\right)$. Let $q \in \mathbb{C}[t]$ generate the conductor of $\mathscr{O}(\tilde{X})=\mathbb{C}[t]$ in $\mathscr{O}(X)$. Then $q \mathscr{D}\left(A^{1}\right) \subset P$ and $P^{*} \subset \mathscr{D}\left(\mathrm{A}^{1}\right) q^{-1}$. Since $\mathscr{D}(X)$ is a Noetherian domain, we can identify $\operatorname{Hom}_{\mathscr{D}\left(\mathrm{A}^{1}\right)}\left(P, \mathscr{D}\left(\mathrm{A}^{1}\right)\right)$ with $P^{*}=\left\{u \in \mathscr{D}(K) \mid u P \subset \mathscr{D}\left(\mathrm{A}^{1}\right)\right\}$. Thus, since $\mathscr{D}\left(A^{1}\right)$ is a hereditary domain,

$$
\text { length }\left(P^{*} / \mathscr{D}\left(\mathrm{A}^{1}\right)\right)=\text { length }\left(\mathscr{D}\left(\mathrm{A}^{1}\right) / P\right) \text {. }
$$

By Lemma 2.6, $\operatorname{dim}_{\mathbb{C}}\left(\mathbb{C}[s, \xi] / \operatorname{gr}_{t} \mathscr{D}(X)\right)<\infty$.

By Proposition $2.4, \mathscr{D}_{0}^{t}(X) \cong \mathrm{gr}_{t} \mathscr{D}(X) \cap \mathbb{C}[\xi]$. Thus

$$
\operatorname{dim}\left(\mathbb{C}[\xi] / \mathscr{D}_{0}^{t}(X)\right)=\operatorname{dim}\left(\mathbb{C}[\xi]+\left(\mathrm{gr}_{t} \mathscr{D}(X)\right) / \mathrm{gr}_{t} \mathscr{D}(X)\right)<\infty
$$

and $\mathscr{D}_{0}^{t}(X)$ is a domain of dimension 1 with integral closure isomorphic to $\mathbb{C}[\xi]$. That $\mathscr{D}_{0}^{t}(X)$ is a finitely generated $\mathbb{C}$-algebra follows from Eakin's Theorem [5, §3.5]. This proves (c) and the theorem.

Combining Theorem 2.7 with Corollary 1.9 yields the theorem stated in the introduction.

THEOREM 2.8. Let $X$ be a curve. Then $\mathcal{N}(X)=\mathscr{O}(X)$ if and only if either (i) $\tilde{X} \neq \mathrm{A}^{1}$ or (ii) $\tilde{X}=\mathrm{A}^{1}$ and $\pi: \tilde{X} \rightarrow X$ is not injective.

Let $X$ be a curve with $\tilde{X}=A^{1}$ and $\pi: \tilde{X} \rightarrow X$ injective. By Theorem 2.7 there exists a curve $Y$ with $\tilde{Y}=A^{1}$ such that $\mathscr{D}_{0}^{t}(X) \cong \mathscr{O}(Y)$. Moreover $\mathscr{O}(Y) \cong \mathscr{D}_{0}^{t}(X)$ is a maximal commutative ad-nilpotent subalgebra of $\mathscr{D}(X)$. It would be interesting to know if there exists a $\mathbb{C}$ algebra isomorphism $\varphi: \mathscr{D}(X) \cong \mathscr{D}(Y)$ such that $\varphi\left(\mathscr{D}_{0}^{t}(X)\right)=\mathscr{O}(Y)$. If this were true then $\varphi(\mathscr{O}(X)) \subset \mathscr{N}(Y) \backslash \mathscr{O}(Y)$, whence $\pi: \tilde{Y} \rightarrow Y$ is injective by Theorem 2.8. In [7] we compute an example where $\mathscr{O}(Y) \not ⿻ \mathscr{O}(X)$ and $\pi: \tilde{Y} \rightarrow Y$ is injective. However, it is unknown if $\mathscr{D}(X) \cong \mathscr{D}(Y)$ for this example. 
3. Non-monomial curves. The remainder of this paper is concerned with those curves $X$ for which $\mathscr{N}(X) \neq \mathscr{O}(X)$. Theorem 2.8 tells us precisely which curves these are. Thus, for the rest of $\S 3$ fix a curve $X$ such that $\tilde{X}=A^{1}$ and $\pi: \tilde{X} \rightarrow X$ is injective. We make the further restriction that $X$ has a unique singularity, which for convenience we take to be $\pi(0)$. Hence if $\mathscr{O}\left(A^{1}\right)=\mathbb{C}[t]$, it follows that $\mathbb{C}[t] \supset \mathscr{O}(X) \supset$ $t^{r} \mathbb{C}[t]$ for some $r$. Our goal is Theorem 3.8 which says that for "most" such $X, \mathscr{D}_{0}^{t}(X) \not \mathscr{O}(X)$.

Definition. A subalgebra $R$ of $\mathbb{C}[t]$ is called a monomial algebra if it has a basis consisting of monomials in $t$ and $\operatorname{dim}_{\mathbb{C}}(\mathbb{C}[t] / R)<\infty$. A curve $X$ is a monomial curve if $\mathscr{O}(X)$ is a monomial algebra. If $R \subset \mathbb{C}[t]$ is a monomial algebra, then $\Lambda=\left\{k \in \mathbb{N} \mid t^{k} \in R\right\}$ is a submonoid of the natural numbers and $R=\sum_{\lambda \in \Lambda} \mathbb{C} t^{\lambda}$. If $R=\mathscr{O}(X)$, call $\Lambda$ the monoid associated to $X$.

The ring of differential operators on a monomial curve has been studied in [6]. Of course $\mathbb{C}[t]$ is a monomial algebra with basis $\{1, t$, $\left.t^{2}, \ldots\right\}$ and thus $A^{1}$ is a monomial curve. A more interesting example is the planar curve $Z$ given by the equation $y^{m}=x^{n}$ where $(m, n)=1$. Then $\mathscr{O}(Z)=\mathbb{C}\left[t^{m}, t^{n}\right]$. Note that a monomial curve $X$ has $\tilde{X}=A^{1}$ and, if $X \neq A^{1}$, unique singularity at $\pi(0)$.

With the above restrictions on $X$, we will show $\mathscr{O}(X) \not \mathscr{D}_{0}^{t}(X)$ if $X$ is not a monomial curve. Let $\left(t^{r}\right)$ be the conductor $\mathscr{O}(\tilde{X})=\mathbb{C}[t]$ in $\mathscr{O}(X)$. Thus $\mathscr{D}(X) t^{r} \subset \mathscr{D}(\tilde{X})=\mathbb{C}[t, \partial]$ so $\mathscr{D}(X) \subset \mathbb{C}\left[t, t^{-1}, \partial\right]$. The element $t \partial \in \mathbb{C}\left[t, t^{-1}, \partial\right]$ is ad-semisimple so $\mathbb{C}\left[t, t^{-1}, \partial\right]$ decomposes into a direct sum of $\operatorname{ad}(t \partial)$-eigenspaces, $\mathbb{C}\left[t, t^{-1}, \partial\right]=\bigoplus_{k \in \mathbb{Z}} t^{k} \mathbb{C}[t \partial]$. Thus $u \in \mathscr{D}(X)$ has a unique expression

$$
u=\sum_{m \leq k \leq l} t^{k} f_{k} \quad \text { with } k \in \mathbb{Z}, f_{k} \in \mathbb{C}[t \partial] \text {, and } f_{l}, f_{m} \neq 0 \text {. }
$$

Call this the standard form for $u$. Give $\mathscr{D}(X)$ the $t$-filtration $\left\{\mathscr{D}_{n}^{t}(X)\right\}_{n \in \mathbb{N}}$ and consider the associated graded ring $\operatorname{gr}_{t} \mathscr{D}(X)$. We will usually apply the following lemma in the case $n=0$.

LEMMA 3.1. If $u \in \mathscr{D}(X)$, write $u=\sum_{m \leq k \leq l} t^{k} f_{k}$ in standard form. Then $u \in \mathscr{D}_{n}^{t}(X)$ if and only if $l \leq n$ and $\operatorname{deg}_{t \partial}\left(f_{k}\right) \leq n-k$ for all $m \leq k \leq l$.

Proof. If $\operatorname{deg}_{t \partial}\left(f_{k}\right) \leq n-k$ for all $m \leq k \leq l$ then $t$-deg $\left(t^{k} f_{k}\right) \leq n$ and $u \in \mathscr{D}_{n}^{t}(X)$. Let $u \in \mathscr{D}_{n}^{t}(X)$ and assume, by way of contradiction, 
that $\left\{k \mid k+\operatorname{deg}\left(f_{k}\right)>n\right\} \neq \varnothing$. Choose $j$ with $s=j+\operatorname{deg}\left(f_{j}\right)>n$ maximal. Note $u$ has a unique expression

$$
u=\sum t^{i} g_{i} \quad \text { with } g_{i} \in \mathbb{C}[\partial] \text { and } i \in \mathbb{Z},
$$

since $u \in \mathbb{C}\left[t, t^{-1}, \partial\right]$, and $g_{i}=0$ for $i>n$ because $t-\operatorname{deg}(u) \leq n$. Set $n(k)=\operatorname{deg}\left(f_{k}\right)$ and note

$$
t^{k} f_{k}=\alpha_{k} t^{k+n(k)} \partial^{n(k)}+(\text { terms of lower degree in both } t \text { and } \partial)
$$

where $0 \neq \alpha_{k} \in \mathbb{C}$. Since $k+n(k) \leq s$ for all $k$,

$$
0=t^{s} g_{s}=\sum\left\{\alpha_{k} t^{k+n(k)} \partial^{n(k)} \mid k+n(k)=s\right\}
$$

Therefore, since $j+n(j)=s$, there exists $i \neq j$ with $i+n(i)=j+n(j)$ and $n(i)=n(j)$. But then $i=j$, a contradiction. Thus $\operatorname{deg}\left(f_{k}\right) \leq n-k$ for $m \leq k \leq l$. Since $\operatorname{deg}\left(f_{l}\right) \geq 0, n-l \geq 0$.

Definition. If $p \in \mathbb{C}[t]$ write $p=\alpha_{0} t^{i}+\alpha_{1} t^{i+1}+\cdots+\alpha_{j} t^{i+j}$ with $\alpha_{0}, \alpha_{j} \neq 0$. Define $\lambda(p)=\alpha_{j} t^{i+j}$ and $\mu(p)=\alpha_{0} t^{i}$. For any curve $Z$ with $\tilde{Z}=A^{1}$, write $\mathscr{O}(\tilde{Z})=\mathbb{C}[t]$ and define

$$
\begin{aligned}
& \Gamma(Z)=\left\{i \in \mathbb{N} \mid \exists q \in \mathscr{O}(Z) \text { with } \lambda(q)=t^{i}\right\}, \\
& \Omega(Z)=\left\{i \in \mathbb{N} \mid \exists q \in \mathscr{O}(Z) \text { with } \mu(q)=t^{i}\right\} .
\end{aligned}
$$

These are both submonoids of $\mathbb{N}$. We write $\Gamma$ for $\Gamma(Z)$ and $\Omega$ for $\Omega(Z)$ if it is clear what curve we mean. If $Z$ is a monomial curve then $\Gamma(Z)$ and $\Omega(Z)$ both equal the monoid associated to $Z$. We have the following converse.

LEMMA 3.2. If $X$ is not a monomial curve then $\Gamma(X) \backslash \Omega(X) \neq \varnothing$.

Proof. Since $X$ is not a monomial curve and $t^{r} \mathbb{C}[t] \subset \mathscr{O}(X)$, we may choose $j \in \Gamma(X)$ maximal such that $t^{j} \notin \mathscr{O}(X)$. We show $j \in$ $\Gamma(X) \backslash \Omega(X)$. If $j \in \Omega(X)$ choose $q \in \mathscr{O}(X)$ of minimal degree such that $\mu(q)=t^{j}$. Then $\lambda(q)=\alpha t^{j+i}$ with $i>0$ and $0 \neq \alpha \in \mathbb{C}$. But $i+j \in \Gamma(X)$ so $t^{i+j} \in \mathscr{O}(X)$ by hypothesis. Hence $p=q-\alpha t^{i+j} \in \mathscr{O}(X)$ with $\mu(p)=t^{j}$, contradicting the minimality of $\operatorname{deg}(q)$.

There is an algebra isomorphism $\varphi: \mathscr{D}_{0}^{t}(X) \cong \mathbb{C}[\xi] \cap \mathrm{gr}_{t} \mathscr{D}(X)$ by Proposition 2.4. By Theorem 2.7, there exists a curve $Y$ with $\tilde{Y}=\mathrm{A}^{1}$ such that $\varphi\left(\mathscr{D}_{0}^{t}(X)\right)=\mathscr{O}(Y) \subset \mathbb{C}[\xi]=\mathscr{O}(\tilde{Y})$. The purpose of the next few results is to compare $\Gamma(Y)$ with $\Omega(X)$ and $\Omega(Y)$ with $\Gamma(X)$. This is done in Proposition 3.7. 
LEMMA 3.3. Let $l \leq 0$ and $u=\sum_{m \leq k \leq l} t^{k} f_{k} \in \mathscr{D}(X)$ in standard form. Then $\prod_{i \in \Gamma \backslash \Gamma-l}(t \partial-i)$ divides $f_{l}$ and $\prod_{i \in \Omega \backslash \Omega-m}(t \partial-i)$ divides $f_{m}$ in $\mathbb{C}[t \partial]$.

Proof. If $i \in \Gamma$ and $(t \partial-i)$ does not divide $f_{l}$, choose $q \in \mathscr{O}(X)$ with $\lambda(q)=t^{i}$. Then $i \in \Gamma-l$ because $u * q \in \mathscr{O}(X)$ and $\lambda(u * q)=f_{l}(i) t^{i+l}$. Thus $\prod_{i \in \Gamma \backslash \Gamma-l}(t \partial-i)$ divides $f_{l}$.

Similarly, if $i \in \Omega$ and $(t \partial-i)$ does not divide $f_{m}$, choose $q \in \mathscr{O}(X)$ with $\mu(q)=t^{i}$. Then $i \in \Omega-m$ since $u * q \in \mathscr{O}(X)$ and $\mu(u * q)=$ $f_{m}(i) t^{i+m}$. Thus $\prod_{i \in \Omega \backslash \Omega-m}(t \partial-i)$ divides $f_{m}$.

LEMMA 3.4. Let $\Lambda$ be a submonoid of $\mathbb{N}$ containing $r+\mathbb{N}$ for some $r \in \mathbb{N}$. If $k \in \mathbb{Z}$, set $n(k)$ equal to the cardinality of the finite set $\Lambda \backslash(\Lambda-k)$. Then $n(-k)=n(k)+k$.

Proof. By symmetry it is enough to prove the result for $k \in \mathbb{N}$. Suppose $k>0$. Partition $\mathbb{N}$ into the disjoint union $\mathbb{N}=\coprod_{i \in \mathbb{N}} \mathbb{N}_{i}$, where $\mathbb{N}_{i}=\{j \in \mathbb{N} \mid i k \leq j<(i+1) k\}$, and set $\Lambda_{i}=\Lambda \cap \mathbb{N}_{i}$. Note $\Lambda \backslash(\Lambda-k)=((\Lambda+k) \backslash \Lambda)-k$. We have

$$
\Lambda \backslash(\Lambda+k)=\coprod_{i \in \mathbb{N}} \Lambda_{i} \backslash\left(\Lambda_{i-1}+k\right)
$$

and

$$
(\Lambda+k) \backslash \Lambda=\coprod_{i \in \mathbb{N}}\left(\Lambda_{i-1}+k\right) \backslash \Lambda_{i}
$$

where $\Lambda_{-1}+k=\varnothing$. Because $r+\mathbb{N} \subset \Lambda$, there exists $N \in \mathbb{N}$ such that $i \geq N$ implies $\Lambda_{i-1}+k=\Lambda_{i}=\mathbb{N}_{i}$. Now

$$
\begin{aligned}
n(-k)-n(k) & =|\Lambda \backslash(\Lambda+k)|-|\Lambda \backslash(\Lambda-k)| \\
& =|\Lambda \backslash(\Lambda+k)|-|(\Lambda+k) \backslash \Lambda| \\
& =\sum_{0 \leq i \leq N}\left|\Lambda_{i} \backslash\left(\Lambda_{i-1}+k\right)\right|-\sum_{1 \leq i \leq N}\left|\left(\Lambda_{i-1}+k\right) \backslash \Lambda_{i}\right| \\
& =\left|\Lambda_{0}\right|+\sum_{1 \leq i \leq N}\left\{\left|\Lambda_{i} \backslash\left(\Lambda_{i-1}+k\right)\right|-\left|\left(\Lambda_{i-1}+k\right) \backslash \Lambda_{i}\right|\right\} \\
& =\left|\Lambda_{0}\right|+\sum_{1 \leq i \leq N}\left\{\left|\Lambda_{i}\right|-\left|\left(\Lambda_{i-1}+k\right)\right|\right\} \\
& =\left|\Lambda_{0}\right|+\sum_{1 \leq i \leq N}\left\{\left|\Lambda_{i}\right|-\left|\Lambda_{i-1}\right|\right\}=\left|\Lambda_{N}\right|=\left|\mathbb{N}_{N}\right|=k
\end{aligned}
$$

Note that we have used the fact that if $A$ and $B$ are finite sets then $|A \backslash B|-|B \backslash A|=|A|-|B|$. 
LEMMA 3.5. Let $m, l \leq 0$ and set $g_{l}=\prod_{i \in \Gamma \backslash \Gamma-l}(t \partial-i)$ and $h_{m}=$ $\prod_{i \in \Omega \backslash \Omega-m}(t \partial-i)$. Then

(a) $\operatorname{deg}_{t \partial}\left(g_{l}\right) \geq-l$ with equality if and only if $-l \in \Gamma$

(b) $\operatorname{deg}_{t \partial}\left(h_{m}\right) \geq-m$ with equality if and only if $-m \in \Omega$.

Proof. Recall that $\Gamma$ and $\Omega$ are both submonoids of $\mathbb{N}$. Thus, by Lemma 3.4, $\operatorname{deg}\left(g_{l}\right)=|\Gamma \backslash(\Gamma-l)|=-l+|\Gamma \backslash(\Gamma+l)| \geq-l$ with equality if and only if $|\Gamma \backslash(\Gamma+l)|=0$. If $-l \in \Gamma$ then $|\Gamma \backslash(\Gamma+l)|=|(\Gamma-l) \backslash \Gamma|=0$ since $\Gamma-l \subset \Gamma$. Conversely, $|(\Gamma-l) \backslash \Gamma|=0$ implies $-l \in \Gamma$ because $0 \in \Gamma$. This proves the assertion for $g_{l}$. The proof for $h_{m}$ is identical.

Proposition 3.6. If $u \in \mathscr{D}_{0}^{t}(X)$ write $u=\sum_{m \leq k \leq l} t^{k} f_{k}$ in standard form. Then $f_{m} \in \mathbb{C} h_{m}$ and $f_{l} \in \mathbb{C} g_{l}$. Moreover, $-l \in \Gamma(X)$ and $-m \in \Omega(X)$.

Proof. By Lemma 3.1, $l \leq 0$ and $\operatorname{deg}\left(f_{k}\right) \leq-k$ for all $k$. By Lemma 3.3, $g_{l}$ divides $f_{l}$. By Lemma 3.5, $\operatorname{deg}\left(g_{l}\right) \geq-l$. Thus $-l \geq \operatorname{deg}\left(f_{l}\right) \geq$ $\operatorname{deg}\left(g_{l}\right) \geq-l$, whence $f_{l} \in \mathbb{C} g_{l} \backslash\{0\}$ and $-l \in \Gamma$ since $\operatorname{deg}\left(g_{l}\right)=-l$. A similar argument works for $f_{m}$.

Proposition 3.7. Write $\varphi\left(\mathscr{D}_{0}^{t}(X)\right)=\mathscr{O}(Y) \subset \mathbb{C}[\xi]$. Then $\Gamma(Y) \subset$ $\Omega(X)$ and $\Omega(Y) \subset \Gamma(X)$.

Proof. Choose $u \in \mathscr{D}_{0}^{t}(X)$. Write $u=\sum_{m \leq k \leq l} t^{k} f_{k}$ in standard form with $l \leq 0$ and $\operatorname{deg}\left(f_{k}\right) \leq-k$. Then

$$
\varphi(u)=\sum\left\{\operatorname{gr}_{t}\left(t^{k} f_{k}\right) \mid \operatorname{deg}\left(f_{k}\right)=-k\right\} \in \mathscr{O}(Y) \subset \mathbb{C}[\xi] .
$$

Thus $\mu(u)=\operatorname{gr}_{t}\left(t^{l} f_{l}\right)=\alpha_{l} \xi^{-l}$, for some $0 \neq \alpha_{l} \in \mathbb{C}$. By Proposition 3.6, $-l \in \Gamma(X)$. Thus $\Omega(Y) \subset \Gamma(X)$. A similar argument shows $\Gamma(Y) \subset \Omega(X)$.

THEOREM 3.8. Let $X$ be a curve with $\tilde{X}=A^{1}, \pi: \tilde{X} \rightarrow X$ injective and unique singularity at $\pi(0)$. If $X$ is not a monomial curve then $\mathscr{D}_{0}^{t}(X)$ is a maximal commutative ad-nilpotent subalgebra of $\mathscr{D}(X)$ which is not isomorphic to $\mathscr{O}(X)$.

Proof. Assume $X$ is not a monomial curve. By Theorem 2.7, $\mathscr{D}_{0}^{t}(X)$ is a maximal commutative ad-nilpotent subalgebra of $\mathscr{D}(X)$ and is isomorphic to $\mathscr{O}(Y)$ for some curve $Y$ with $\tilde{Y}=A^{1}$. If there exists $\vartheta: \mathscr{O}(X) \cong \mathscr{O}(Y)$ then, since $\mathbb{C}[t]$ is the integral closure of both 
$\mathscr{O}(X)$ and $\mathscr{O}(Y), \vartheta$ extends to an automorphism of $\mathbb{C}[t]$. Thus $\vartheta(t)=$ $\alpha t+\beta$ for some $\alpha, \beta \in \mathbb{C}$ with $\alpha \neq 0$. By Lemma 3.2, there exists $i \in \Gamma(X) \backslash \Omega(X)$. Choose $q \in \mathscr{O}(X)$ with $\lambda(q)=t^{i}$. Then $\lambda \vartheta(q)=$ $\alpha^{i} t^{i}$, whence $i \in \Gamma(Y)$. But $\Gamma(Y) \subset \Omega(X)$ by Proposition 3.7, a contradiction.

The results in this section were motivated by the explicit computation, given in [7], of $\mathscr{D}(X)$ when $\mathscr{O}(X)=\mathbb{C} \oplus \mathbb{C}\left(t^{2}+t^{3}\right) \oplus t^{4} \mathbb{C}[t]$. In this case $\mathscr{D}_{0}^{t}(X)$ is isomorphic to the ring of regular functions on a curve with two singularities, while $X$ has only one.

4. Monomial curves. Recall that $\mathscr{D}_{0}^{t}\left(\mathrm{~A}^{1}\right)=\mathbb{C}[\partial]$ and that there exists an automorphism of $\mathscr{D}\left(\mathrm{A}^{1}\right)$ which interchanges $t$ and $-\partial$. For a monomial curve $X$ we will see, in this section, that $\mathscr{D}_{0}^{t}(X) \cong \mathscr{O}(X)$. In general, however, there is no automorphism of $\mathscr{D}(X)$ interchanging $\mathscr{O}(X)$ and $\mathscr{D}_{0}^{t}(X)$ when $X$ is a monomial curve. This is proved in [7]. Also in this section it is proved that for all but two monomial curves $X$ there exists a maximal commutative ad-nilpotent subalgebra of $\mathscr{D}(X)$ which is not isomorphic to $\mathscr{O}(X)$. Indeed, this subalgebra is not isomorphic to any monomial algebra.

Throughout $\S 4$, let $X$ be a monomial curve. Hence $\mathscr{D}(X) \subset$ $\mathbb{C}\left[t, t^{-1}, \partial\right]$ and, since $\mathscr{O}(X)$ is spanned by monomials, $t \partial \in \mathscr{D}(X)$. Thus $\mathscr{D}(X)$ is an $\operatorname{ad}(t \partial)$-stable subspace of $\mathbb{C}\left[t, t^{-1}, \partial\right]$, whence $\mathscr{D}(X)=$ $\bigoplus_{k \in \mathbb{Z}}\left\{t^{k} \mathbb{C}[t \partial] \cap \mathscr{D}(X)\right\}$. If $u \in \mathscr{D}(X)$ and $f \in \mathscr{O}(X)$, recall that $u * f$ denotes the action of $u$ on $f$. The following lemma is implicit in [6].

LEMMA 4.1. If $X$ is a monomial curve with associated monoid $\Lambda$, then $\mathscr{D}(X)=\bigoplus_{k \in \mathbb{Z}} t^{k} f_{k} \mathbb{C}[t \partial]$ with $f_{k}=\Pi\{t \partial-j \mid j \in \Lambda \backslash(\Lambda-k)\} \in$ $\mathbb{C}[t \partial]$.

Proof. By the above paragraph, $\mathscr{D}(X)=\bigoplus_{k \in \mathbb{Z}}\left\{t^{k} \mathbb{C}[t \partial] \cap \mathscr{D}(X)\right\}$. But $\mathscr{D}(X) \cap t^{k} \mathbb{C}[t \partial]$ is a $\mathbb{C}[t \partial]$-submodule of $t^{k} \mathbb{C}[t \partial]$. Since $t^{k} \mathbb{C}[t \partial] \cong$ $\mathbb{C}[t \partial]$ as $\mathbb{C}[t \partial]$-module and $\mathbb{C}[t \partial]$ is a PID, $t^{k} \mathbb{C}[t \partial] \cap \mathscr{D}(X)=t^{k} f_{k} \mathbb{C}[t \partial]$ for some $f_{k} \in \mathbb{C}[t \partial]$. Set $h_{k}=\Pi\{t \partial-j \mid j \in \Lambda \backslash(\Lambda-k)\}$. If $g \in$ $\mathbb{C}[t \partial]$ then $t^{k} g * t^{n}=g(n) t^{n+k}$. Using this it is easy to check that $t^{k} h_{k} * \mathscr{O}(X) \subset \mathscr{O}(X)$, whence $t^{k} h_{k} \in \mathscr{D}(X)$. Let $t^{k} g \in \mathscr{D}(X)$ and $j \in \Lambda$. If $g(j) \neq 0$ then $g(j) t^{k+j}=t^{k} g * t^{j} \in \mathscr{O}(X)$ so $k+j \in \Lambda$. This implies $h_{k}$ divides $g$ in $\mathbb{C}[t \partial]$ and thus $f_{k}=h_{k}$.

THEOREM 4.2. If $X$ is a monomial curve with associated monoid $\Lambda$ and $\mathscr{D}(X)=\bigoplus_{k \in \mathbb{Z}} t^{k} f_{k} \mathbb{C}[t \partial]$ then $\mathscr{D}_{0}^{t}(X)=\sum_{i \in \Lambda} \mathbb{C} t^{-i} f_{-i}$. In particular, $\mathscr{D}_{0}^{t}(X) \cong \mathscr{O}(X)$. 
Proof. Let $u=\sum t^{k} p_{k} \in \mathscr{D}_{0}^{t}(X)$, with $p_{k} \in \mathbb{C}[t \partial]$. Note $\Lambda=\Gamma(X)=$ $\Omega(X)$ so, in the notation of Proposition 3.5, $f_{k}=h_{k}=g_{k}$. Since $X$ is a monomial curve, $t^{k} p_{k} \in \mathscr{D}(X)$ for all $k$. Thus $t-\operatorname{deg}\left(t^{k} p_{k}\right) \geq 0$. But, by Lemma 3.1, $t-\operatorname{deg}\left(t^{k} p_{k}\right) \leq 0$. Thus $t-\operatorname{deg}\left(t^{k} p_{k}\right)=0$ so, if $p_{k} \neq 0$, $-k \in \Lambda$ and $p_{k}=g_{k}=f_{k}$ by Proposition 3.6. Of course, if $-k \in \Lambda$ then $t^{-k} f_{-k}=t^{-k} g_{-k} \in \mathscr{D}_{0}^{t}(X)$.

By Proposition $2.4, \mathscr{D}_{0}^{t}(X) \cong \mathrm{gr}_{t} \mathscr{D}_{0}^{t}(X)$ as $\mathbb{C}$-algebras. Combining this with the observation that $\operatorname{gr}_{t}\left(t^{-k} f_{-k}\right)=\xi^{k}$ for $k \in \Lambda$ gives the second statement from the first.

Combining Theorems 4.2 and 3.8 yields the following.

Corollary 4.3. Let $X$ be a curve with $\tilde{X}=A^{1}, \pi: \tilde{X} \rightarrow X$ injective and unique singularity at $\pi(0)$. Then $\mathscr{D}_{0}^{t}(X) \cong \mathscr{O}(X)$ if and only if $X$ is a monomial curve.

The next results show that, for all but two monomial curves, there exists a maximal commutative ad-nilpotent subalgebra of $\mathscr{D}(X)$ not isomorphic to $\mathscr{O}(X)$. For $v \in \mathscr{D}(K)$, define ord $(v)$ to be the order of $v$ as a differential operator, the " $\partial$-degree". If $R$ is any ring and $u \in R$ is locally ad-nilpotent we may consider, as in [1], $\exp (\operatorname{ad}(u)) \in \operatorname{Aut}(R)$.

Lemma 4.4. Set $\Phi=\exp \left(\operatorname{ad}\left(\lambda t^{k}\right)\right) \in \operatorname{Aut}\left(\mathbb{C}\left[t, t^{-1}, \partial\right]\right)$ where $\lambda \in \mathbb{C}$ and $k \in \mathbb{Z}$. Let $X$ be a curve with $\tilde{X}=A^{1}, \pi: \tilde{X} \rightarrow X$ injective and unique singularity at $\pi(0)$. If $R=\Phi \mathscr{D}(X)$ then $\operatorname{gr}_{\partial} R=\operatorname{gr}_{\partial} \mathscr{D}(X)$.

Proof. If $u \in \mathscr{D}(X)$, let $\operatorname{ord}(u)=n$ and write $u=f \partial^{n}+v$ with $f \in \mathbb{C}[t] \backslash\{0\}, v \in \mathbb{C}\left[t, t^{-1}, \partial\right]$ and $\operatorname{ord}(v)<n$. Since $\operatorname{ord}\left(\left[\lambda t^{k}, w\right]\right)<$ $\operatorname{ord}(w)$ for all $w \in \mathscr{D}(K)$, ord $\Phi(v)<\operatorname{ord} \Phi\left(f \partial^{n}\right)$ and

$$
\begin{aligned}
\Phi(u) & =\Phi\left(f \partial^{n}\right)+\Phi(v) \\
& =f \partial^{n}+\left(\sum_{i \geq 1}(i !)^{-1} \operatorname{ad}\left(\lambda t^{k}\right)^{i}\left(f \partial^{n}\right)\right)+\Phi(v)=f \partial^{n}+y
\end{aligned}
$$

where $y \in \mathbb{C}\left[t, t^{-1}, \partial\right]$ and $\operatorname{ord}(y)<n$. Thus $\operatorname{gr}_{\partial}(\Phi(u))=f \xi^{n}=$ $\operatorname{gr}_{\partial}(u)$.

Proposition 4.5. Set $\Phi=\exp \left(\operatorname{ad}\left(\lambda t^{k}\right)\right) \in \operatorname{Aut}\left(\mathbb{C}\left[t, t^{-1}, \partial\right]\right)$, where $\lambda \in \mathbb{C}$ and $k \in \mathbb{N}$. Let $X$ be a curve with $\tilde{X}=\mathrm{A}^{1}, \pi: \tilde{X} \rightarrow X$ injective and unique singularity at $\pi(0)$. Set $R_{0}$ equal to the 0 th part of the $t$-filtration on $R=\Phi \mathscr{D}(X) \subset \mathbb{C}\left[t, t^{-1}, \partial\right]$. Then $R_{0}$ is a maximal 
commutative ad-nilpotent subalgebra of $R$. Moreover, $R_{0}$ is a finitely generated domain with integral closure isomorphic to $\mathbb{C}[\xi]$.

Proof. Lemma 4.4 implies $\mathrm{gr}_{\partial} R \subset \mathbb{C}[t, \xi]$. Thus, by Proposition 2.4, $\mathrm{gr}_{t} R \subset \mathbb{C}[s, \xi]$ and $R_{0}$ is isomorphic to $\left(\mathrm{gr}_{t} R\right) \cap \mathbb{C}[\xi]$. Moreover, $R_{0}$ is a maximal commutative ad-nilpotent subalgebra of $R$ if $R_{0} \neq \mathbb{C}$. Set $P=\Phi \mathscr{D}(X, \tilde{X}), Q=\Phi \mathscr{D}(\tilde{X}, X)$ and let $q \in \mathbb{C}[t]$ generate the conductor of $\mathbb{C}[t]$ in $\mathscr{O}(X)$. Then length $(P / \mathbb{C}[t, \partial])=$ length $(\mathbb{C}[t, \partial] / Q)$ as $\mathbb{C}[t, \partial]$-modules because $\Phi \in \operatorname{Aut}(\mathbb{C}[t, \partial])$. Furthermore

$$
Q P=\Phi(\mathscr{D}(\tilde{X}, X) \mathscr{D}(X, \tilde{X}))=\Phi \mathscr{D}(X)=R .
$$

Since $\Phi$ fixes $\mathbb{C}(t)$ and

$$
\mathbb{C}[t, \partial] q^{-1} \supset \mathscr{D}(X, \tilde{X}) \supset \mathbb{C}[t, \partial] \supset \mathscr{D}(\tilde{X}, X) \supset q \mathbb{C}[t, \partial],
$$

we have

$$
\mathbb{C}[t, \partial] q^{-1} \supset P \supset \mathbb{C}[t, \partial] \supset Q \supset q \mathbb{C}[t, \partial] .
$$

Thus by Lemma 2.6, $\operatorname{dim}_{\mathbb{C}}\left(\mathbb{C}[s, \xi] / \operatorname{gr}_{t} R\right)<\infty$ and $\left(\mathrm{gr}_{t} R\right) \cap \mathbb{C}[\xi] \cong R_{0}$ is of finite codimension in $\mathbb{C}[\xi]$. The rest follows as in the proof of Theorem 2.7.

Let $X$ be a monomial curve with associated monoid $\Lambda$. Write $\mathscr{D}(X)=\bigoplus_{k \in \mathbb{Z}} t^{k} f_{k} \mathbb{C}[t \partial]$ with $f_{k} \in \mathbb{C}[t \partial]$. If $\Lambda \cup\{1\} \neq \mathbb{N}$, we show $\mathscr{D}(X)$ contains a maximal commutative ad-nilpotent subalgebra which is not isomorphic to any monomial algebra. Given $m \in \mathbb{N} \backslash(\Lambda \cup\{1\})$, set $\Phi=\exp \left(\operatorname{ad}\left(m^{-1} t^{m}\right)\right)$ and $R=\Phi(\mathscr{D}(X)) \subset \mathbb{C}\left[t, t^{-1}, \partial\right]$. Then $R_{0}$, the 0 th part of the $t$-filtration on $R$, is a maximal commutative ad-nilpotent subalgebra of $R$ by Proposition 4.5. Moreover, $R_{0} \cong$ $\mathbb{C}[\xi] \cap \mathrm{gr}_{t}(R)$. Note that $\Phi^{-1}=\exp \left(\operatorname{ad}\left(-m^{-1} t^{m}\right)\right)$.

LeMMA 4.6. Let $\Psi=\exp \left(\operatorname{ad}\left(-k^{-1} t^{k}\right)\right) \in$ Aut $\mathbb{C}\left[t, t^{-1}, \partial\right]$ with $0 \neq$ $k \in \mathbb{N}$. If $n \in \mathbb{Z}$ and $\alpha_{1}, \ldots, \alpha_{r} \in \mathbb{C}$ then there exist $h_{0}, \ldots, h_{r} \in \mathbb{C}[t \partial]$ such that

$$
\Psi\left(t^{n} \prod_{1 \leq i \leq r}\left(t \partial-\alpha_{i}\right)\right)=t^{n} \sum_{0 \leq j \leq r} t^{j k} h_{j} .
$$

Moreover, the leading term of $h_{j}$ is $\left(\begin{array}{c}r \\ j\end{array}\right)(t \partial)^{r-j}$ and $h_{0}=\prod_{1 \leq i \leq r}\left(t \partial-\alpha_{i}\right)$. 
P. PERKINS

Proof.

$$
\begin{aligned}
& \Psi\left(t^{n} \prod_{1 \leq i \leq r}\left(t \partial-\alpha_{i}\right)\right) \\
& \quad=\Psi\left(t^{n}\right) \Psi\left(\prod_{1 \leq i \leq r}\left(t \partial-\alpha_{i}\right)\right)=t^{n} \prod_{1 \leq i \leq r}\left(\left(t \partial-\alpha_{i}\right)+t^{k}\right) .
\end{aligned}
$$

The result follows by expanding the product using $\left(t \partial-\alpha_{i}\right) t^{l}=$ $t^{l}\left(t \partial-\alpha_{i}+l\right)$. For example,

$$
t^{k} h_{1}=\left(t \partial-\alpha_{1}\right) \cdots\left(t \partial-\alpha_{r-1}\right) t^{k}+\cdots+t^{k}\left(t \partial-\alpha_{2}\right) \cdots\left(t \partial-\alpha_{r}\right) .
$$

LEMMA 4.7. If $0 \neq n \in \mathbb{N}$ and $f \in \mathbb{C}[t \partial] \backslash\{0\}$ then $t^{-n} f \notin R_{0}$.

Proof. Assume $t^{-n} f \in R_{0}$ and note $\operatorname{deg}(f)=n$. Then $\Phi^{-1}\left(t^{-n} f\right)=$ $t^{-n} \sum_{0 \leq j \leq n} t^{j m} h_{j}$ with $h_{0}=f$, by Lemma 4.6. Since $X$ is a monomial curve, $t^{-n} f=t^{-n} h_{0} \in \mathscr{D}_{0}^{t}(X)$ and so $f=f_{-n}$ with $n \in \Lambda$. Thus write

$$
f_{-n}=\prod\{t \partial-j \mid j \in \Lambda \backslash(\Lambda+n)\}=\prod_{1 \leq i \leq n}\left(t \partial-\alpha_{i}\right) \quad \text { with } \alpha_{n}=0 .
$$

Now $t^{m-n} h_{1} \in \mathscr{D}(X)$ and

$$
\begin{aligned}
t^{m} h_{1}= & {\left[\left(t \partial-\alpha_{1}\right) \cdots\left(t \partial-\alpha_{n-1}\right) t^{m}\right] } \\
& +\left[\left(t \partial-\alpha_{1}\right) \cdots\left(t \partial-\alpha_{n-2}\right) t^{m}\left(t \partial-\alpha_{n}\right)\right] \\
& +\cdots+\left[t^{m}\left(t \partial-\alpha_{2}\right) \cdots\left(t \partial-\alpha_{n}\right)\right] \\
= & t^{m}\left\{\left[\left(t \partial-\alpha_{1}+m\right) \cdots\left(t \partial-\alpha_{n-1}+m\right)\right]\right. \\
& \quad+\left[\left(t \partial-\alpha_{1}+m\right) \cdots\left(t \partial-\alpha_{n-2}+m\right)\left(t \partial-\alpha_{n}\right)\right]+\ldots \\
& \left.\quad+\left[\left(t \partial-\alpha_{2}\right) \cdots\left(t \partial-\alpha_{n}\right)\right]\right\} .
\end{aligned}
$$

Since $\alpha_{n}=0,\left\{\prod_{1<i \leq n-1}\left(-\alpha_{i}+m\right)\right\} t^{m-n}=t^{m-n} h_{1} * 1 \in \mathscr{O}(X)$. Since $m \notin \Lambda, \prod_{1 \leq i \leq n-1}\left(-\alpha_{i}+m\right) \neq 0$. Thus $m-n \in \Lambda$ and $n \notin \Lambda$, a contradiction.

Lemma 4.8. Let $u=\sum_{0 \leq k \leq n} t^{-k} g_{k} \in R_{0}$ be in standard form. Then $v=\sum_{l \geq 0} t^{l m-n} g_{n-l m} \in R_{0}$ also.

Proof. Let $r(k)=\operatorname{deg}\left(g_{k}\right)$. By Lemma 4.6, set

$$
\Phi^{-1}\left(t^{-k} g_{k}\right)=t^{-k} \sum_{0 \leq i \leq r(k)} t^{i m} h_{k, i}
$$


for each $k$. Then

$$
\Phi^{-1}(u)=\sum_{0 \leq k \leq n} \sum_{0 \leq i \leq r(k)} t^{i m-k} h_{k, i}=\sum_{s \geq-n} t^{s} \sum_{i m-k=s} h_{k, i} .
$$

By Lemma 4.1, $t^{s} \sum_{i m-k=s} h_{k, i} \in \mathscr{D}(X)$ for each $s$. For $k \leq n, i m-k=$ $j m-n$ if and only if $k=n-l m$ for some $l \in \mathbb{N}$. Thus

$$
\boldsymbol{\Phi}^{-1}(v)=\sum_{j \geq 0} t^{j m-n} \sum\left\{h_{k, i} \mid i m-k=j m-n\right\} \in \mathscr{D}(X) .
$$

Thus $v \in \Phi \mathscr{D}(X)=R$ and the result follows.

LEMMA 4.9. If $R_{0}$ is isomorphic to $\mathscr{D}(X)$ then for every $n \in \Lambda$ there exists $u \in R_{0}$ such that $u=\sum_{0 \leq i \leq n} t^{-i} g_{i}$, in standard form, with $g_{n} \neq 0$.

Proof. Consider both $R_{0}$ and $\mathscr{O}(X)$ as subalgebras of $\mathbb{C}[\xi]$ of finite co-dimension. If $\vartheta: \mathscr{O}(X) \cong R_{0}$ then $\vartheta$ extends to an automorphism of $\mathbb{C}[\xi]$. Thus $\vartheta\left(\xi^{n}\right)=(a \xi+b)^{n}$ and there exists $u \in R_{0}$ such that $\operatorname{gr}_{t}(u)=a^{n} \xi^{n}+\left(\right.$ lower degree terms). Write $u=\sum_{k \leq i \leq l} t^{-i} g_{i}$, in standard form, with $g_{l} \neq 0$. Then $l \geq n$ and to prove that $l=n$ it suffices to show that $t-\operatorname{deg}\left(t^{-l} g_{l}\right)=0$. By Lemma 3.1, $t-\operatorname{deg}\left(t^{-i} g_{i}\right) \leq 0$ for all $i$. In particular $k \geq 0$. As in the proof of Lemma 4.8, if we write

$$
\Phi^{-1}(u)=\sum_{s \geq-l} t^{s} \sum_{i m-k=s} h_{k, i}
$$

then $t^{-l} g_{l}=t^{-l} \sum_{i m-k=-l} h_{k, i} \in \mathscr{D}(X)$. By Proposition 2.4, $\mathrm{gr}_{t} \mathscr{D}(X)$ $\subset \mathbb{C}[t, \xi]$, from which it follows that $t-\operatorname{deg}\left(t^{-l} g_{l}\right) \geq 0$.

THEOREM 4.10. Let $X$ be a monomial curve with associated monoid $\Lambda$ such that $\Lambda \cup\{1\} \neq \mathbb{N}$. Then $\mathscr{D}(X)$ contains a maximal commutative ad-nilpotent subalgebra which is not isomorphic to $\mathscr{O}(X)$.

Proof. Assume there exists an isomorphism $\vartheta: \mathscr{O}(X) \rightarrow R_{0}$. Suppose there exists $0 \neq n \in \Lambda$ such that $n<m$. By Lemma 4.9 there exists $\sum_{0 \leq i \leq n} t^{-i} g_{i} \in R_{0}$, in standard form, with $g_{n} \neq 0$. Since $l m-n>0$ for all $l \in \mathbb{N}$, Lemma 4.8 implies $t^{-n} g_{n} \in R_{0}$, contradicting Lemma 4.7. Thus we may assume $\Lambda=\{0\} \cup(\mathbb{N}+r)$ for some $r \geq 3$ and take $m=r-1$. By Lemma 4.9, there exists $u \in R_{0}$ such that $u=\sum_{0 \leq i \leq r} t^{-i} g_{i}$ in standard form with $g_{r} \neq 0$. By Lemma 4.8, take $u=t^{-r} g_{r}+t^{-1} g_{1}$ where $\operatorname{deg}\left(g_{1}\right) \leq 1$.

Make the identification $R_{0} \cong \mathrm{gr}_{t}\left(R_{0}\right)$ and consider $R_{0}$ and $\mathscr{O}(X)$ as subalgebras of $\mathbb{C}[\xi]$. As in the proof of Lemma 4.9, $\vartheta$ extends to an 
automorphism of $\mathbb{C}[\xi]$. If $\operatorname{deg}\left(g_{1}\right)=1$ then $\operatorname{gr}_{t}(u)=c \xi^{r}+d \xi$ with $c, d \in \mathbb{C} \backslash\{0\}$. If $v=\vartheta^{-1}(u) \in \mathscr{O}(X)$ then $v=e \xi^{r}+f$ for some $e, f \in \mathbb{C}$, since $\vartheta\left(\xi^{n}\right)=(a \xi+b)^{n}$. But $r>2$ so,

$$
\vartheta(v)=e(a \xi+b)^{r}+f \neq c \xi^{r}+d \xi=\mathrm{gr}_{t}(u) .
$$

Thus $\operatorname{deg}\left(g_{1}\right)=0$ and $u=t^{-r} g_{r}+\alpha t^{-1}$ for some $\alpha \in \mathbb{C}$.

We have $t^{-r} g_{r} \in \mathscr{D}(X)$, as in the proof of Lemma 4.9, and hence $g_{r}=\Pi\{t \partial-j \mid j \in \Lambda \backslash(\Lambda+r)\}$ by Lemma 4.2. Note that $2 m=2(r-1) \in$ $\Lambda \backslash(\Lambda+r)$. As in the proof of Lemma 4.6,

$$
\begin{aligned}
\Phi^{-1}(u) & =\Phi^{-1}\left(t^{-r} g_{r}\right)+\Phi^{-1}\left(\alpha t^{-1}\right) \\
& =t^{-r} \Phi^{-1}\left(g_{r}\right)+\alpha t^{-1} \\
& =t^{-r} \Pi\left\{t \partial-j+t^{m} \mid j \in \Lambda \backslash(\Lambda+r)\right\}+\alpha t^{-1} \\
& =t^{-r} \sum_{0 \leq i \leq r} t^{i m} h_{i}+\alpha t^{-1}
\end{aligned}
$$

for some $h_{i} \in \mathbb{C}[t \partial]$. Factor $g_{r}=\left(t \partial-\alpha_{1}\right) \cdots\left(t \partial-\alpha_{r}\right)$ with $\alpha_{r}=0$ and $\alpha_{1}=2 m$ and note $t^{r-2} h_{2}=t^{-r+2 m} h_{2} \in \mathscr{D}(X)$. Now

$$
\begin{aligned}
t^{2 m} h_{2}= & \left(t \partial-\alpha_{1}\right) \cdots\left(t \partial-\alpha_{r-2}\right) t^{2 m} \\
& +\left(t \partial-\alpha_{1}\right) \cdots\left(t \partial-\alpha_{r-3}\right) t^{m}\left(t \partial-\alpha_{r-1}\right) t^{m} \\
& +\cdots+t^{2 m}\left(t \partial-\alpha_{2}\right) \cdots\left(t \partial-\alpha_{r}\right) \\
= & t^{2 m}\left[\left(t \partial-\alpha_{1}+2 m\right) \cdots\left(t \partial-\alpha_{r-2}+2 m\right)\right. \\
& \quad+\left(t \partial-\alpha_{1}+2 m\right) \cdots\left(t \partial-\alpha_{r-3}+2 m\right)\left(t \partial-\alpha_{r-1}+m\right) \\
& \left.\quad+\cdots+\left(t \partial-\alpha_{2}\right) \cdots\left(t \partial-\alpha_{r}\right)\right] .
\end{aligned}
$$

The only summand of $h_{2}$ which does not have a factor of $t \partial$, in the form of $t \partial-\alpha_{r}$ or $t \partial-\alpha_{1}+2 m$, is $\left(t \partial-\alpha_{2}+m\right) \cdots\left(t \partial-\alpha_{r-1}+m\right)$. Thus

$$
\left(-\alpha_{2}+m\right) \cdots\left(-\alpha_{r-1}+m\right) t^{r-2}=t^{r-2} h_{2} * 1 \in \mathscr{O}(X) .
$$

Since $m \notin \Lambda$ and $\alpha_{i} \in \Lambda$ for all $i,\left(-\alpha_{2}+m\right) \cdots\left(-\alpha_{r-1}+m\right) \neq 0$. But $r-2 \notin \Lambda$, a contradiction.

The only monomial curves with $\Lambda \cup\{1\}=\mathbb{N}$ and $A^{1}$ and $y^{2}=x^{3}$. In [1], it is shown that all maximal commutative subalgebras of $\mathscr{D}\left(A^{1}\right)$, contained in $\mathscr{N}\left(\mathrm{A}^{1}\right)$, are isomorphic to $\mathscr{O}\left(\mathrm{A}^{1}\right)=\mathbb{C}[t]$. Mimicking this proof, the same is shown for $y^{2}=x^{3}$ in [7]. Hence $y^{2}=x^{3}$ is the only curve with normalization equal to $A^{1}, \pi$ injective and unique singularity at $\pi(0)$ such that all maximal commutative ad-nilpotent subalgebras of its ring of differential operators are isomorphic. 
5. Simply connected curves. This section is devoted to proving the following proposition, referred to in the introduction.

Proposition 5.1. Let $X$ be a curve. Then $\pi_{1}(X)=0$ if and only if $\tilde{X}=\mathrm{A}^{1}$ and $\pi: \tilde{X} \rightarrow X$ is injective.

Proof. Note that $\pi_{1}\left(A^{1}\right)=0$ and $\pi$ is continuous in the usual topology. Assume $\tilde{X}=A^{1}$ and $\pi: \tilde{X} \rightarrow X$ is injective, hence bijective. Thus $\pi$ is a homomorphism and induces an isomorphism between $\pi_{1}(X)$ and $\pi_{1}\left(A^{1}\right)$. Therefore $\pi_{1}(X)=0$.

Assume $\pi_{1}(X)=0$. We first show $\pi$ is injective and $\pi_{1}(\tilde{X})=0$. Recall $\pi$ identifies at most a finite number of points. Factor $\pi$ into a sequence of maps

$$
\tilde{X} \rightarrow Y_{0} \rightarrow Y_{1} \rightarrow \cdots \rightarrow Y_{n}=X
$$

where $\tilde{X} \rightarrow Y_{0}$ is injective and $Y_{i} \rightarrow Y_{i+1}$ identifies just two points. Thus consider the map $A \rightarrow B$ where $A$ equals some $Y_{i}$ and $B$ is $A$ modulo the identification of two points $x, y \in A$. Without affecting the homotopy of $A$, we may draw out two thin "whiskers" at points $x$ and $y$ and, since $A$ is path connected, assume they both originate at the same point. Thus $B$ is homotopically equivalent to $A \vee S^{1}$, the one point union of $A$ with a circle. By a standard application of Van Kampen's Theorem [4], it follows that $\pi_{1}(B) \cong \pi_{1}(A) * \mathbb{Z}$, the free product. By induction, $\pi_{1}(X) \cong \pi_{1}(\tilde{X}) * \mathbb{Z} * \cdots * \mathbb{Z}$, where there are $n$ copies of $\mathbb{Z}$. Thus $\pi_{1}(\tilde{X})=0$ and $n=0$, whence $\pi$ is injective.

To see $\tilde{X}=A^{1}$, we first show genus $(\tilde{X})=0$. Let $Z$ be the nonsingular projective model for $\tilde{X}$. Then $Z$ is a complex nonsingular projective curve and hence is homeomorphic to a compact Riemann surface. It is enough to see that $\operatorname{genus}(Z)=0$. Since $\tilde{X}$ equals $Z$ less a finite number of points, there exists a series of inclusions

$$
\tilde{X}=Y_{0} \subset Y_{1} \subset \cdots \subset Y_{n}=Z
$$

where $Y_{i}$ is $Y_{i+1}$, less one point. Another easy application of Van Kampen's theorem implies that $\pi_{1}\left(Y_{i+1}\right)$ is a homomorphic image of $\pi_{1}\left(Y_{i}\right)$. Since $\pi_{1}(\tilde{X})=0$ we have $\pi_{1}(Z)=0$, by induction. Hence $Z=\mathbb{P}^{1}$ and genus $(Z)=0$. Thus genus $(\tilde{X})=0$, whence $\tilde{X}$ equals $A^{1}$ less a finite number of points. But $\pi_{1}(\tilde{X})=0$, so $\tilde{X}=A^{1}$. 


\section{REFERENCES}

[1] J. Dixmier, Sur les algèbres de Weyl, Bull. Soc. Math. France, 96 (1968), 209242.

[2] T. Levasseur, S. P. Smith and J. T. Stafford, The minimal nilpotent orbit, the Joseph ideal and differential operators, J. Algebra, (to appear).

[3] L. Makar-Limanov, Rings of differential operators on algebraic curves, (to appear).

[4] W. S. Massey, Algebraic Topology: An Introduction, Harcourt, Brace and World, Inc., New York, 1967.

[5] H. Matsumura, Commutative Algebra, W. A. Benjamin Co., New York, 1970.

[6] I. M. Musson, Some rings of differential operators which are Morita equivalent to the Weyl algebra $A_{1}$, Proc. Amer. Math. Soc., 98 (1986), 29-30.

[7] P. Perkins, Commutative Subalgebras of the Ring of Differential Operators on a Curve, University of Washington, (1988), (Thesis).

[8] S. P. Smith and J. T. Stafford, Differential operators on an affine curve, Proc. London Math. Soc., (3) 56 (1988), 229-259.

[9] J. T. Stafford, Endomorphisms of right ideals of the Weyl algebra, Trans. Amer. Math. Soc., 299 (1987), 623-639.

Received March 1, 1988.

UNIVERSITY OF WASHINGTON

SEATTLE, WA 98195 